PREPARED FOR THE U.S. DEPARTMENT OF ENERGY, UNDER CONTRACT DE-AC02-76CH03073

PPPL-3911

PPPL-3911

UC-70

Resistive Drift Waves in a Bumpy Torus

by

J.L.V. Lewandowski

January 2004

N/M|

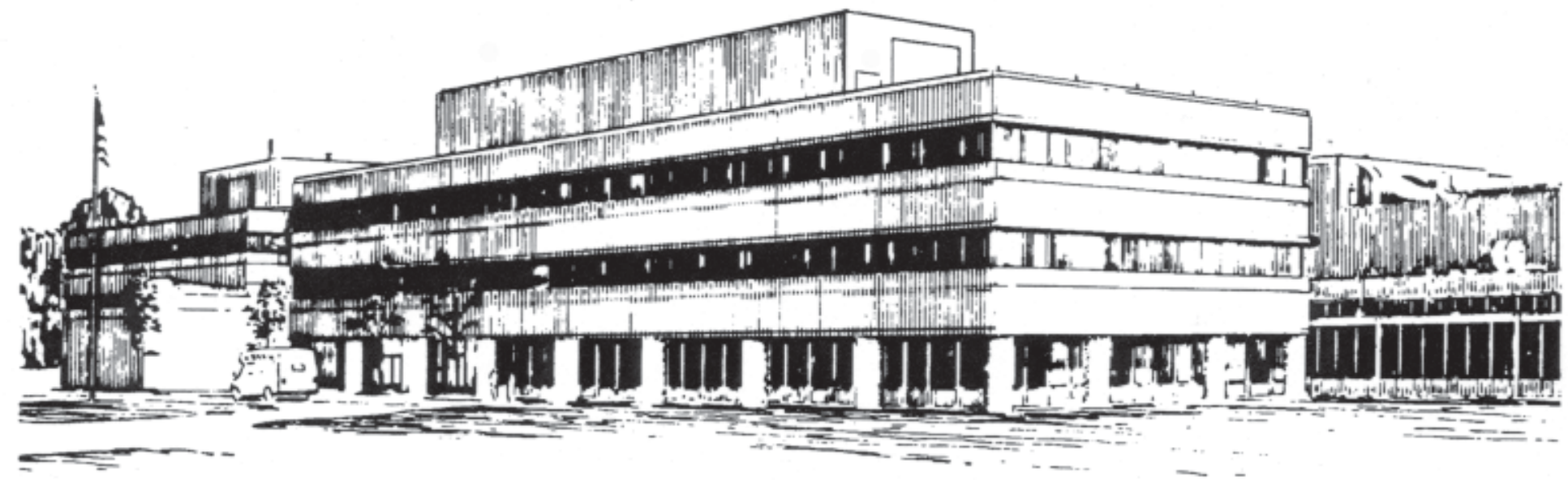

PRINCETON PLASMA PHYSICS LABORATORY PRINCETON UNIVERSITY, PRINCETON, NEW JERSEY 


\section{PPPL Reports Disclaimer}

This report was prepared as an account of work sponsored by an agency of the United States Government. Neither the United States Government nor any agency thereof, nor any of their employees, makes any warranty, express or implied, or assumes any legal liability or responsibility for the accuracy, completeness, or usefulness of any information, apparatus, product, or process disclosed, or represents that its use would not infringe privately owned rights. Reference herein to any specific commercial product, process, or service by trade name, trademark, manufacturer, or otherwise, does not necessarily constitute or imply its endorsement, recommendation, or favoring by the United States Government or any agency thereof. The views and opinions of authors expressed herein do not necessarily state or reflect those of the United States Government or any agency thereof.

\section{Availability}

This report is posted on the U.S. Department of Energy's Princeton Plasma Physics Laboratory Publications and Reports web site in Fiscal Year 2004. The home page for PPPL Reports and Publications is: http://www.pppl.gov/pub_report/

DOE and DOE Contractors can obtain copies of this report from:

U.S. Department of Energy

Office of Scientific and Technical Information

DOE Technical Information Services (DTIS)

P.O. Box 62

Oak Ridge, TN 37831

Telephone: (865) 576-8401

Fax: (865) 576-5728

Email: reports@adonis.osti.gov

This report is available to the general public from:

National Technical Information Service

U.S. Department of Commerce

5285 Port Royal Road

Springfield, VA 22161

Telephone: $1-800-553-6847$ or

(703) $605-6000$

Fax: (703) 321-8547

Internet: http://www.ntis.gov/ordering.htm 


\title{
Resistive Drift Waves in a Bumpy Torus
}

\author{
J.L.V. Lewandowski * \\ Princeton Plasma Physics Laboratory \\ Princeton University, P.O. Box 451 \\ Princeton NJ 08543 \\ USA
}

December 22, 2003

\begin{abstract}
A computational study of resistive drift waves in the edge plasma of a bumpy torus is presented. The magnetohydrodynamic equilibrium is obtained from a three-dimensional local equilibrium model. The use of a local magnetohydrodynamic equilibrium model allows for a computationally-efficient systematic study of the impact of the magnetic field structure on drift wave stability.
\end{abstract}

Pacs \# : 52.35Kt, 52.30Jb, 52.35Ra

\section{Introduction}

There is growing evidence that slow, drift-type modes, known as drift waves, are responsible for a substantial part of the observed anomalous transport in tokamak and stellarator plasmas [1]. Drift waves represent a special class of gradient instabilities which are driven unstable by a source of free energy in the density and/or temperature gradients. In order to determine the linear properties of drift waves (and other drift-type modes satisfying $k_{\|} / k_{\perp} \ll 1$, where $k_{\|}$ and $k_{\perp}$ are the parallel and perpendicular wavevectors, respectively) in toroidal geometry, the model equations can be solved using the ballooning representation of Connor et al [2] as an eigenvalue [8] or as initial-value problem [7] for a set of representative field lines. Although some

*e-mail: jlewando@pppl.gov 
understanding of the drift wave dynamics can be gained using the so-called $i \delta$ model (for which the parameter $\delta$ is used as a tuning parameter for the drive of the instability), more realistic models usually require the solution of two or more coupled partial differential equations to be solved on a given field line. A natural approach for solving such systems of equations is to use an initial-value algorithm; this method also ensures that the fastest growing mode is (numerically) observed.

Although the topic of drift wave stability and dynamics in tokamak plasmas has been theoretically and numerically studied quite extensively, the study of low-frequency drift-type modes in stellarator geometry has received much less attention. One major reason for this state of affairs is that stellarator plasmas are inherently three-dimensional and usually require the use of sophisticated equilibrium codes to specify their magnetohydrodynamic (MHD) equilibria. As mentioned above, the most stringent linear stability considerations are usually based on the eikonal representation for perturbations; the problem is then reduced to an initial-value (or eigenvalue) problem along the magnetic field line; in general, the linear stability properties are studied one magnetic surface at a time. This observation is one motivation behind the threedimensional local magnetohydrodynamic of Hegna [3]. Hegna's equilibrium model is particularly efficient for drift wave calculations as the MHD equilibrium is determined one surface at a time; this low-cost method allows us to study the effect of magnetic surface shaping (or parameterization) on drift wave stability. Although the initial parameterization of the magnetic surface in the local MHD equilibrium model can be quite general [see Eq.(5)], the main focus of this paper is a stellarator with an helical magnetic axis.

This paper is organized as follows; in section 2, the simplest self-consistent model equations governing resistive drift wave in a collisional plasma are presented. The characterization of the local MHD equilibrium is given in section 3. Section 4 describes the numerical method used to solve the equations governing the two-field resistive model. Numerical results are presented in section 5, and conclusions are given in section 6

\section{Resistive Drift Wave Model}

We consider drift waves in a low-temperature, high-density edge plasma. In a typical edge plasma, the electron-ion collision frequency can be high enough to prevent the electrons from responding instantaneously to the perturbed electrostatic potential; as a result, the nonadiabatic electron response does not vanish and the background density gradient feeds the unstable drift mode. The most unstable modes are strongly elongated along the direction of the equilibrium magnetic field $\left(k_{\|} / k_{\perp} \ll 1\right)$ and it is convenient to use the eikonal representation for each fluctuating quantity $\tilde{f}$

$$
\widetilde{f}=\widehat{f} \exp \left(i \epsilon^{-1} S\right),
$$

where $\alpha=\theta-\iota \zeta$ is the field line label, $\theta$ is the magnetic poloidal angle, $\zeta$ is the magnetic toroidal angle, $\iota$ is the rotational transform, $S$ is the eikonal and $\epsilon$ is a smallness parameter. Here $\widehat{f}(\psi, \alpha, \theta)$ is the (slowly-varying) envelope (or amplitude) and $\nabla S \sim \nabla \widehat{f}=\mathcal{O}(1)$. As drift waves are characterized by short perpendicular wavelength and long parallel wavelength, one can demand that the eikonal $S$ satisfies the relation of $\mathbf{B} \cdot \boldsymbol{\nabla} S=0$ which implies that $S=S(\alpha, \psi)$. Noting that $\partial \widehat{f} / \partial \psi \ll \epsilon^{-1} \partial S / \partial \psi$ and that $\partial \widehat{f} / \partial \alpha \ll \epsilon^{-1} \partial S / \partial \alpha$, these quantities can be neglected 
to this order. We can then write

$$
\widetilde{f}=\widehat{f}(\theta) \exp \left[i \epsilon^{-1} S(\psi, \alpha)\right],
$$

By analogy with the Fourier representation, the perpendiculat wave number can be defined as

$$
\begin{aligned}
\mathbf{k}_{\perp} & \equiv-i \tilde{f}^{-1} \nabla_{\perp} \tilde{f}=\epsilon^{-1} \nabla_{\perp} S=\epsilon^{-1} \frac{\partial S}{\partial \alpha}\left(\nabla \alpha+\frac{\partial S / \partial \psi}{\partial S / \partial \alpha} \nabla \psi\right) \\
& =\epsilon^{-1} \frac{\partial S}{\partial \alpha}\left[\nabla \theta-\iota \nabla \zeta-\left(\zeta \frac{d \iota}{d \psi}-\frac{\partial S / \partial \psi}{\partial S / \partial \alpha}\right) \boldsymbol{\nabla} \psi\right] .
\end{aligned}
$$

Our model equations [See Eqs. $(2,3)]$ are to be solved along a given field line labeled by $\alpha$ on a flux surface $\psi$. This field line passes through the point $\left(\theta_{0}, \zeta_{0}\right)$. The equilibrium is periodic and therefore invariant under the operations

$$
\alpha\left(\psi, \theta_{0}, \zeta_{0}\right) \mapsto \alpha\left(\psi, \theta_{0}+2 \pi, \zeta_{0}\right) \text { and } \alpha\left(\psi, \theta_{0}, \zeta_{0}\right) \mapsto \alpha\left(\psi, \theta_{0}, \zeta_{0}+2 \pi\right) \text {. }
$$

The perpendicular wavevector, $\mathbf{k}_{\perp}$, defined above must fulfil the same periodicity constraints. The periodicity constraints are satisfied if we require

$$
\frac{\partial S / \partial \psi}{\partial S / \partial \alpha}=\left(\zeta_{0}+\zeta_{k}\right) \frac{d \iota}{d \psi}
$$

where $\zeta_{k}$ is a free parameter for radially local studies. Using the field line equation, $\theta-\iota \zeta=$ $\theta_{0}-\iota \zeta_{0}$, one can write the perpendicular wavevector as

$$
\mathbf{k}_{\perp}=\epsilon^{-1} \frac{\partial S}{\partial \alpha}\left[\nabla \theta-\iota \nabla \zeta-\left(\frac{\theta-\theta_{0}}{\iota}-\zeta_{k}\right) \frac{d \iota}{d \psi} \nabla \psi\right]
$$

In a low- $\beta$, cold-ion plasma, the model equations governing resistive drift wave (see Appendix A for details) are: the quasineutrality equation

$$
\frac{1}{\bar{B}^{2}} \frac{\partial \bar{\omega}}{\partial \bar{t}}=2 \xi \bar{B} L_{n} \nabla_{\|}\left(\frac{L_{n}}{\bar{B}} \nabla_{\|} \widetilde{h}\right)-2 \mathbf{Q} \cdot \frac{\widehat{\mathbf{b}} \times \rho_{\mathrm{s} 0} \nabla \widetilde{n}}{\bar{B}},
$$

and the electron continuity equation

$$
\frac{\partial \widetilde{n}}{\partial \bar{t}}=\nabla \rho \cdot \frac{\widehat{\mathbf{b}} \times \rho_{\mathrm{s} 0} \nabla \widetilde{\Phi}}{\bar{B}}-2 \mathbf{Q} \cdot \frac{\widehat{\mathbf{b}} \times \rho_{\mathrm{s} 0} \nabla \widetilde{h}}{\bar{B}}+2 \xi \bar{B} L_{n} \nabla_{\|}\left(\frac{L_{n}}{\bar{B}} \nabla_{\|} \widetilde{h}\right)
$$

where $\bar{\omega}=\rho_{\mathrm{s} 0}^{2} \nabla_{\perp}^{2} \widetilde{\Phi}$ and $\xi \equiv \omega_{\star} \tau_{e}\left(m_{i} / m_{e}\right) \gg 1$ is termed the collisional parameter; $\omega_{\star}=$ $\sqrt{T_{e} / m_{i}} / L_{n}$ is the drift frequency, $L_{n}$ is the density scalelength, $\mathbf{Q} \equiv L_{n} \nabla B / B$ is related to the curvature of the magnetic field and $\rho_{\mathrm{s} 0}=\sqrt{T_{e} / m_{i}} /\left(e B_{0} / m_{i} c\right) ; \bar{B} \equiv B / B_{0}$ where $B_{0}$ is a magnetic field of reference (see next section); finally $\widetilde{h}=\widetilde{n}-\widetilde{\Phi}$ is the nonadiabatic response of the electrons. The normalized time is $\bar{t}=\omega_{\star} t$ where $\omega_{\star}$ is the drift frequency. Here $\rho=\rho(\psi)$ is a radial coordinate satisfying $\mathbf{B} \cdot \nabla \rho=0$ which is defined in the next section. The terms involving $\mathbf{Q}$ in Eqs.(2,3) arise because the divergence of the $\mathbf{E} \times \mathbf{B}$ drift velocity and the divergence of the electron diamagnetic flux do not vanish in a sheared $\mathbf{B}$ field. The first term on the righthand side of Eq.(3) is the free energy, contained in the background density gradient, that drives the drift wave. As it is evident in Eqs. $(2,3)$, the key parameter is the collisional parameter, $\xi$, which is inversely proportional to the collision frequency: in presence of collisions, the electrons cannot achieve perfect adiabicity along the field lines and the drift mode can become unstable. In practice, Eqs. $(2,3)$ are solved as an initial-value problem for the amplitudes $\widehat{\Phi}$ and $\widehat{n}$. The MHD equilibrium quantities that enter Eqs. $(2,3)$ are determined in the next section. 


\section{Local magnetohydrodynamic equilibrium}

The confining magnetic field is written in straight-field line coordinates

$$
\mathbf{B}=\nabla \psi \times \nabla \alpha
$$

where $\alpha \equiv \theta-\iota \zeta$ is the field line label, $\theta$ is the magnetic poloidal angle, $\zeta$ is the magnetic toroidal angle, $\iota$ is the rotational transform, and $\psi$ is related to the magnetic toroidal flux. Without loss of generality, one can specify the shape of the magnetic surface in terms of the cylindrical coordinates $(R, \phi, Z)$ as

$$
\begin{aligned}
R & =\sum_{m=0}^{M} \sum_{n=-N}^{N} R_{m n} \cos \left(\varphi_{m n}\right) \\
\phi & =-\zeta+\sum_{m=0}^{M} \sum_{n=-N}^{N} \phi_{m n} \sin \left(\varphi_{m n}\right), \\
Z & =\sum_{m=0}^{M} \sum_{n=-N}^{N} Z_{m n} \sin \left(\varphi_{m n}\right),
\end{aligned}
$$

where $\varphi_{m n} \equiv m \theta+n N_{p} \zeta, N_{p}$ is the number of field periods, and the poloidal and toroidal Fourier mode numbers $M$ and $N$, as well as the amplitudes $\left\{R_{m n}, \phi_{m n}, Z_{m n}\right\}$, are free parameters. Using the parameterization (5) one can determine the covariant basis vectors $\mathbf{e}_{\theta}=\partial \mathbf{r} / \partial \theta$ and $\mathbf{e}_{\zeta}=\partial \mathbf{r} / \partial \zeta$, where $\mathbf{r}$ is the position vector, and the metric elements $g_{\theta \theta}=\mathbf{e}_{\theta} \cdot \mathbf{e}_{\theta}, g_{\theta \zeta}=\mathbf{e}_{\theta} \cdot \mathbf{e}_{\zeta}$

and $g_{\zeta \zeta}=\mathbf{e}_{\zeta} \cdot \mathbf{e}_{\zeta}$. It is convenient to define an orthonormal vector set $\{\widehat{\mathbf{b}}, \widehat{\mathbf{n}}, \widehat{\mathbf{g}}\}$ attached to the magnetic field lines

$$
\begin{array}{rlrl}
\widehat{\mathbf{b}} & \equiv \frac{\mathbf{B}}{B}=\frac{\mathbf{e}_{\eta}}{\left|\mathbf{e}_{\eta}\right|} & & \text { (parallel) } \\
\widehat{\mathbf{n}} & \equiv \frac{\mathbf{e}_{\theta} \times \mathbf{e}_{\zeta}}{\left|\mathbf{e}_{\theta} \times \mathbf{e}_{\zeta}\right|} & \text { (normal) } \\
\widehat{\mathbf{g}} & \equiv \widehat{\mathbf{b}} \times \widehat{\mathbf{n}} & \text { (geodesic) }
\end{array}
$$

where $\mathbf{e}_{\eta} \equiv \mathbf{e}_{\zeta}+\iota \mathbf{e}_{\theta}$. The vector set $\{\widehat{\mathbf{b}}, \widehat{\mathbf{n}}, \widehat{\mathbf{g}}\}$ can be determined from the parameterization (5). Finally, one can calculate important geometrical attributes of the confining $\mathbf{B}$ field such as the geodesic curvature, $\kappa_{g}=\widehat{\mathbf{g}} \cdot[(\widehat{\mathbf{b}} \cdot \nabla) \widehat{\mathbf{b}}]$, the normal curvature, $\kappa_{n}=\widehat{\mathbf{n}} \cdot[(\widehat{\mathbf{b}} \cdot \nabla) \widehat{\mathbf{b}}]$, and the normal torsion $\tau_{n}=-\widehat{\mathbf{n}} \cdot[(\widehat{\mathbf{b}} \cdot \boldsymbol{\nabla}) \widehat{\mathbf{g}}]$. In order to determine the Jacobian of the transformation, $\mathcal{J}=[\boldsymbol{\nabla} \psi \cdot(\boldsymbol{\nabla} \theta \times \boldsymbol{\nabla} \zeta)]^{-1}=\mathbf{e}_{\psi} \cdot\left(\mathbf{e}_{\theta} \times \mathbf{e}_{\zeta}\right)$, we demand that the normal current vanishes everywhere on the magnetic surface [3]; using Ampere's law, one then has $J_{n} \equiv \widehat{\mathbf{n}} \cdot \mathbf{J} \propto \nabla \psi \cdot(\boldsymbol{\nabla} \times \mathbf{B}) \equiv 0$, or

$$
\frac{\partial}{\partial \theta}\left(\frac{F(\theta, \zeta)}{\partial}\right)=\frac{\partial}{\partial \zeta}\left(\frac{G(\theta, \zeta)}{\partial}\right),
$$

where

$$
\begin{aligned}
& F(\theta, \zeta)=g_{\zeta \zeta}+\iota g_{\theta \zeta} \\
& G(\theta, \zeta)=g_{\theta \zeta}+\iota g_{\theta \theta}
\end{aligned}
$$


Eq.(7) introduces the primary constraint on the choice $\mathbf{r}=\mathbf{r}(\theta, \zeta)$ [Eq.(5)]. Eq.(7) is termed the Jacobian constraint. Note that in the axisymmetric case, the Jacobian constraint admits the exact solution of

$$
\mathcal{J}=f(\psi) F(\theta, \zeta)
$$

where $f(\psi)$ is an arbitrary flux surface quantity. In the general case, Eq.(7) must be solved numerically. Assuming that a solution has been found, one can construct $\boldsymbol{\nabla} \psi=\left(\mathbf{e}_{\theta} \times \mathbf{e}_{\zeta}\right) / \mathcal{J}$ and $\mathbf{B}=\mathbf{e}_{\eta} / \mathcal{J}$. The next step consists in calcultating the parallel current density consistent with the radial force balance equation and the quasineutrality condition. Using the radial force balance equation

$$
\mathbf{J} \times \mathbf{B}=c \boldsymbol{\nabla} p
$$

in the quasineutrality condition, $\boldsymbol{\nabla} \cdot \mathbf{J}=0$, one obtains

$$
\boldsymbol{\nabla} \cdot \mathbf{J}_{\|}=-\boldsymbol{\nabla} \cdot \mathbf{J}_{\perp}=2 c \frac{d p}{d \psi} \frac{|\nabla \psi|}{B} \kappa_{g}
$$

where the Jacobian constraint, Eq.(7), has been used. Substituting $\sigma \equiv \mathbf{J} \cdot \mathbf{B} / B^{2}=\langle\sigma\rangle+\widehat{\sigma}$ in Eq.(10), one arrives at the equation of

$$
\mathbf{B} \cdot \nabla \lambda=2 \frac{|\nabla \psi|}{B} \kappa_{g}
$$

where $\lambda \equiv \widehat{\sigma} /\left(c p^{\prime}\right)$ (a prime denotes a derivative with respect to the toroidal flux function) and the quantity $|\nabla \psi| / B$ can be calculated directly from the parameterization (5)

$$
\frac{|\nabla \psi|}{B}=\sqrt{\frac{g_{\theta \theta} g_{\zeta \zeta}-g_{\theta \zeta}^{2}}{g_{\zeta \zeta}+2 \iota g_{\theta \zeta}+\iota^{2} g_{\theta \theta}}} .
$$

The flux surface quantity $\langle\sigma\rangle$ is yet to be determined. The flux surface average of any function $F$ is defined as

$$
\langle F\rangle \equiv \frac{\int_{0}^{2 \pi} d \zeta \int_{0}^{2 \pi} d \theta \mathcal{J} F}{\int_{0}^{2 \pi} d \zeta \int_{0}^{2 \pi} d \theta \mathcal{J}}=\frac{1}{V^{\prime}} \int_{0}^{2 \pi} d \zeta \int_{0}^{2 \pi} d \theta \mathcal{J} F
$$

where $V$ is the plasma volume enclosed within $\psi$. Note that if $\mathcal{J}$ is a solution of Eq.(7), then $f(\psi) \mathcal{J}$ is also a solution of the same equation. Since the arbitrary flux surface function $f(\psi)$ is not known, the quantity $V^{\prime}$ is left undetermined; in practice, $V^{\prime}$ is used as an overall normalization factor. The flux surface quantity $\langle\sigma\rangle$ can be obtained through the local magnetic shear defined as $S \equiv \widehat{\mathrm{g}} \cdot \boldsymbol{\nabla} \times \widehat{\mathrm{g}}$, which can also be written as (Appendix B)

$$
S=\frac{|\nabla \psi|^{2}}{B^{2}} \mathbf{B} \cdot \boldsymbol{\nabla}\left(D+\zeta \frac{d \iota}{d \psi}\right)
$$

where

$$
D \equiv \frac{\iota \nabla \zeta \cdot \nabla \psi-\nabla \theta \cdot \nabla \psi}{\nabla \psi \cdot \nabla \psi}
$$


Using $\mathbf{B} \cdot \boldsymbol{\nabla} \zeta=1 / \mathcal{J}$ and noting that the flux surface average operator, $\langle\bullet\rangle$, annihilates the $\mathbf{B} \cdot \boldsymbol{\nabla}$ operator, we get from Eq.(14)

$$
\left\langle\frac{S B^{2}}{|\boldsymbol{\nabla} \psi|^{2}}\right\rangle=4 \pi^{2} \frac{\iota^{\prime}}{V^{\prime}}
$$

Substituting $S=4 \pi J_{\|} /(c B)-2 \tau_{n}$ in the left-hand side of Eq.(16) and noting that $\langle\widehat{\sigma}\rangle \equiv 0$, one obtains

$$
\langle\sigma\rangle=c \sigma_{0}-c \sigma_{1} \frac{d p}{d \psi}-c \sigma_{2} \frac{d \iota}{d \psi},
$$

where $\sigma_{0}=C_{3} /\left(2 \pi C_{1}\right), \sigma_{1}=C_{2} / C_{1}$ and $\sigma_{2}=\pi / C_{1}$; we have defined

$$
\begin{aligned}
& C_{1}=\int_{0}^{2 \pi} d \zeta \int_{0}^{2 \pi} \frac{B^{2} \mathcal{\partial}}{g^{\psi \psi}} d \theta \\
& C_{2}=\int_{0}^{2 \pi} d \zeta \int_{0}^{2 \pi} \frac{B^{2} \jmath \partial}{g^{\psi \psi}} d \theta
\end{aligned}
$$

and

$$
C_{3}=\int_{0}^{2 \pi} d \zeta \int_{0}^{2 \pi} \frac{B^{2} \partial \tau_{n}}{g^{\psi \psi}} d \theta
$$

In summary, given the parameterization (5), one solves Eq.(7) for the Jacobian, followed by Eq.(11) for $\lambda$ (which is proportional to the part of $J_{\|} / B$ that varies in the magnetic surface). Given $\iota^{\prime}$ and $p^{\prime}$ (free parameters) one calculates $\langle\sigma\rangle$ through Eq.(17) and the specification of the local MHD equilibrum is complete.

\section{Numerical Method}

In this section, the numerical method used to solve the two-field resistive drift wave model is presented. The motivation for using a semi-implicit algorithm is discussed.

The radial coordinate is conveniently defined as $\rho \equiv R_{0} \sqrt{2 \bar{\psi}}$ where $\bar{\psi} \equiv \psi / \psi_{0}$ and $\psi_{0}=$ $B_{0} R_{0}^{2}$ and $R_{0} \equiv R_{00}$ is the major radius [in the large-aspect ratio tokamak equilibrium, $\psi \simeq$ $B_{0} r^{2} / 2$ where $B_{0}$ is the magnetic field strength evaluated at the magnetic axis; it follows that $\rho=r$ in this case]. In the general 3D case, it is convenient to define a magnetic field strength of reference as $B_{0} \equiv\left\langle B^{2}\right\rangle^{1 / 2}$. Using the eikonal representation [Eq.(1)] in the quasineutrality condition [Eq.(2)] and the electron continuity equation [Eq.(3)] we obtain

$$
\frac{\partial \widehat{\Phi}}{\partial \bar{t}}=-\eta D_{\|}-i \eta \nu_{\perp} \widehat{n}
$$

and

$$
\frac{\partial \widehat{n}}{\partial \bar{t}}=-i \sqrt{b} \widehat{\Phi}+D_{\|}+i \nu_{\perp}(\widehat{n}-\widehat{\Phi})
$$


where

$$
D_{\|}=\nu_{\|} \bar{B} S_{\|} \frac{\partial}{\partial \bar{\theta}}\left(\frac{S_{\|}}{\bar{B}} \frac{\partial(\widehat{n}-\widehat{\Phi})}{\partial \bar{\theta}}\right) .
$$

Here $S_{\|}(\bar{\theta})=1 /(\bar{\partial} \bar{B}), \bar{\partial}=\left[R_{0} \nabla \bar{\psi} \cdot\left(R_{0} \nabla \theta \times R_{0} \boldsymbol{\nabla} \zeta\right)\right]^{-1}$ is the dimensionless Jacobian and $\nu_{\|}=$ $2 \xi \epsilon_{n}^{2} / q^{2}$ is related to the parallel transport of current density. The perpendicular transport is controlled by the curvature term

$$
\nu_{\perp}(\bar{\theta}) \equiv 2 \sqrt{b} \epsilon \epsilon_{n} S_{\perp}(\bar{\theta})
$$

which arises from the divergence of the electron diamagnetic flux and the divergence of the $\mathbf{E} \times \mathbf{B}$ drift velocity. In the above equations, we have defined $b \equiv\left(k_{\theta} \rho_{\mathrm{s} 0}\right)^{2}, k_{\theta} \equiv N_{0} / \bar{a}$ is the characteristic perpendicular wavevector (i.e. $\left.|\nabla \alpha|_{\bar{\theta}=0}^{2} \sim k_{\theta}^{2}\right), \epsilon_{n} \equiv L_{n} / R_{0}$ and $\epsilon \equiv \bar{a} / R_{0}$ is the inverse aspect ratio. The geometrical quantities $\mathcal{L}$ and $S_{\perp}$ are defined as

$$
\begin{gathered}
\mathcal{L}(\bar{\theta}) \equiv \frac{1+\Lambda^{2}}{R_{0}^{2} g^{\overline{\psi \psi}}}, \\
S_{\perp}(\bar{\theta}) \equiv \frac{\kappa_{n}+\Lambda \kappa_{g}}{\sqrt{g \overline{\psi \psi}}},
\end{gathered}
$$

and

$$
\Lambda(\bar{\theta}) \equiv-\frac{\nabla \psi \cdot \nabla \alpha}{B}
$$

Finally $\eta(\bar{\theta}) \equiv 1 /\left(b \epsilon^{2} \mathcal{L}\right) \simeq 1 / \bar{\theta}^{2}$ for large $|\bar{\theta}|$. In view of the secular behavior of the polarization term $\left(\mathcal{L} \sim \bar{\theta}^{2}\right.$ for $\left.|\bar{\theta}| \gg 1\right)$, it is numerically convenient to solve for the nonadiabatic response $\widehat{h} \equiv \widehat{n}-\widehat{\Phi}$ instead of solving the quasineutrality equation (18). The system of equations to be solved can then be written as

$$
\frac{\partial \widehat{n}}{\partial t}=-i \sqrt{b} \widehat{\Phi}+D_{\|}+i \nu_{\perp} \widehat{h}
$$

and

$$
\frac{\partial \widehat{h}}{\partial t}=-i\left(\sqrt{b}+\nu_{\perp}\right) \widehat{\Phi}+(1+\eta) D_{\|}+i(1+\eta) \nu_{\perp} \widehat{n}
$$

Eqs. $(25,26)$ are discretized on the domain $\bar{\theta} \in\left[-\bar{\theta}_{\max },+\bar{\theta}_{\max }\right]$ where $\bar{\theta}_{\max }$ is a free parameter. In a low-temperature, high-density edge plasma the collisional parameter is much larger than unity [typically $\xi=\mathcal{O}\left(10^{2}\right)$ ] and one expects the parallel transport to be strong. Therefore a numerical method based on an explicit scheme will require a very small time step. The time step for an explicit method is constrained by the transport along the magnetic field lines and must be less than the well-known Courant-Friedrichs-Lewy (CFL) criterion [4] of

$$
\Delta t<(\Delta t)_{\mathrm{CFL}} \equiv \frac{q^{2}(\Delta \bar{\theta})^{2}}{2 \xi \epsilon_{n}^{2}},
$$


where $\Delta \bar{\theta}$ is the grid spacing. In practise, the grid spacing $\Delta \bar{\theta}$ must be chosen small enough as to capture the details of the equilibrium along the magnetic field line. In order to bypass the stringent condition (27) we must resort to an implicit scheme. As it turns out, for the system of Eqs. $(25,26)$, it is sufficient to use a semi-implicit numerical scheme; this is done by treating $\partial \widehat{n} / \partial \bar{t}$ and $\partial \widehat{h} / \partial \bar{t}$ and the parallel transport terms [terms in $D_{\|}$in Eq. $(25,26)$ ] implicitly whereas the remaining terms are treated explicitly. As a result one obtains a set of coupled tridiagonal systems which can be solved sequentially using standard algorithms, such as the Thomas algorithm [6].

In order to determine the mode frequency, $\omega$, we use the transformation of

$$
\widehat{\Phi}(\bar{\theta}, t) \mapsto \bar{\Phi}(\bar{\theta}) \exp (-i \omega t) .
$$

The normal mode frequency can be written as $\omega=\Re(\omega)+i \Im(\omega) \equiv \omega_{r}+i \gamma$. Noting that

$$
\frac{\partial \widehat{\Phi}}{\partial t}=\frac{1}{2} \frac{1}{|\widehat{\Phi}|} \frac{\partial}{\partial t}|\widehat{\Phi}|^{2}-i \omega_{r}|\widehat{\Phi}|
$$

we obtain

$$
\gamma=\frac{1}{\left|\langle\widehat{\Phi}\rangle_{\bar{\theta}}\right|} \frac{\partial}{\partial t}\left|\langle\widehat{\Phi}\rangle_{\bar{\theta}}\right|,
$$

for the linear growth rate and

$$
\omega_{r}=-\Im\left(\frac{1}{\langle\widehat{\Phi}\rangle_{\bar{\theta}}} \frac{\partial}{\partial t}\langle\widehat{\Phi}\rangle_{\bar{\theta}}\right),
$$

for the mode frequency. Equation (28) is also denoted $\gamma_{\Phi}$ below. The linear growth rate associated with the perturbed plasma density is given

$$
\gamma_{n}=\frac{1}{\left|\langle\widehat{n}\rangle_{\bar{\theta}}\right|} \frac{\partial}{\partial t}\left|\langle\widehat{n}\rangle_{\bar{\theta}}\right| .
$$

In the above equations, we have defined the field-line average operator as

$$
\langle F\rangle_{\bar{\theta}} \equiv \frac{1}{2 \bar{\theta}_{\max }} \int_{-\bar{\theta}_{\max }}^{+\bar{\theta}_{\max }} F\left(\bar{\theta}^{\prime}, t\right) d \bar{\theta}^{\prime},
$$

for any function $F(\bar{\theta}, t)$. The free parameter in Eq.(31) must be large enough so that the linear growth rate [Eq.(28)] and the mode frequency [Eq.(29)] become independent of $\bar{\theta}_{\max }$; a convergence study using this parameter is presented in the next section.

\section{$5 \quad$ Numerical Results}

As described in section 3, the specification of the local MHD equilibrium depends on 2 free parameters, $\iota^{\prime}$ and $p^{\prime}$. It is convenient to use normalized quantities instead of $\iota^{\prime}$ and $p^{\prime}$; to make 
the connection with the standard large-aspect ratio tokamak notation, we define the ballooning parameter

$$
\alpha_{b} \equiv-\frac{2}{\pi^{2} \iota^{2}} \frac{V^{\prime}}{\left\langle\frac{B^{2}}{g^{\psi \psi}}\right\rangle^{1 / 2}} \frac{d p}{d \psi}
$$

and the global shear parameter

$$
s_{b} \equiv-\frac{4 \pi^{2} R_{0}}{V^{\prime}} \frac{\frac{1}{\iota} \frac{d \iota}{d \psi}}{\left\langle\frac{B^{2}}{g^{\psi \psi}}\right\rangle} .
$$

As mentioned in the Introduction, the initial parameterization of the magnetic surface [Eq.(5)] is quite general. However, in order to illustrate the usefulness and efficiency of the local equilibrium model for drift wave stability calculations, we consider the parameterization of

$$
\begin{aligned}
R & =R_{0}\left[1+\epsilon_{\mathrm{t}} \cos \theta+\epsilon_{\mathrm{h}} \cos (N \zeta)\right] \\
\phi & =-\zeta \\
Z & =R_{0}\left[\epsilon_{\mathrm{t}} \sin \theta+\epsilon_{\mathrm{h}} \cos (N \zeta)\right]
\end{aligned}
$$

where $N \equiv N_{p}$ is the number of field periods; $\epsilon_{\mathrm{t}}$ and $\epsilon_{\mathrm{h}}$ are termed the toroidicity parameter and the helical parameter, respectively. In the remaining of this paper, we refer to Eq.(34) as the helical parameterization. Note that the case $\epsilon_{\mathrm{h}}=0$ in Eq.(34) corresponds to the tokamak parameterization with concentric, circular magnetic surfaces (which is valid for a low- $\beta$ plasma).

In all simulations reported in this paper, the initial profile for $\widehat{\Phi}$ has been chosen to be a Gaussian profile with its maxima centered at $\bar{\theta}=0$. The density profile at $t=0$ is chosen as $\widehat{n}=\widehat{\Phi}$, that is the nonadiabatic response vanishes at $t=0$; as a result, the parallel transport terms in Eqs. $(25,26)$, which involve $D_{\|}$, vanish at the beginning of the simulations. Since the cross-field terms in Eqs. $(25,26)$ are not equal (even when $\widehat{n}=\widehat{\Phi}$ ), the non-adiabatic response becomes nonzero as time progresses. As the instability develops, the parallel transport increases in order to balance the cross-field transport; at this stage, the linear growth rate becomes almost independent of time (Figure 4). In these studies, the linear growth rates associated with the perturbed electrostatic potential and plasma density have been maximized with respect to the parameter $\zeta_{k}$. For the specific cases, it has been found that the fastest linear growth rate, $\gamma_{\ell}$, is the largest at $\zeta_{k}=0$ and that $\gamma_{\ell}$ decreases weakly with increasing $\zeta_{k}$. The numerical study has been carried for the field line that passes through the outboard side of the torus $\left(\zeta_{0}=\theta_{0}=0\right)$; this choice has been motivated by the fact that the normal curvature is strongly unfavorable there. The numerical study can of course be repeated for various field lines.

The real (plain) and imaginary (dotted) parts of the normalized electrostatic potential amplitude at $\omega_{\star} t=10$ is shown in Figure 2. The parameters are: $\alpha_{b}=0$ (ballooning parameter), $s_{b}=0.25$ (global shear parameter), $q=2.28$ (safety factor), $n_{0}=5 \times 10^{12} \mathrm{~cm}^{-3}$ (equilibrium plasma density), $T_{e}=5 \mathrm{eV}$ (equilibrium electron temperature), $k_{\theta} \rho_{\mathrm{s} 0}=0.8, B_{0}=10^{4}$ Gauss (equilibrium magnetic field of reference), $\Delta \bar{\theta}=\pi / 128$ (grid spacing), $L_{n}=4.0 \mathrm{~cm}$ (density scalelength), $R_{0}=10^{2} \mathrm{~cm}$ (major radius), $\epsilon_{\mathrm{t}}=0.2$ (toroidicity parameter) and $\epsilon_{\mathrm{h}}=0.1$ (helical parameter). The number of field periods is $N=3$. Figure 3 shows the corresponding profile for the perturbed density amplitude. We note that the fluctuating electrostatic potential and the 
plasma density are out of phase and that their respective amplitudes are not equal indicating that the drift mode is unstable. The collision parameter for Figures 2 and 3 is $\xi=71.6$.

Figure 4 shows the instantaneous linear growth rate as a function of the normalized time $\bar{t}=\omega_{\star} t$ for the electrostatic potential (plain line) and the perturbed plasma density (dotted line). The parameters are the same as those of Figures 2 and 3. After transitory effects, the growth rates reach a common asymptotic value after a few growth times; a growth time is defined as the reciprocal of the average linear growth rate, $\tau \equiv 1 / \gamma_{\ell}$, where $\gamma_{\ell}=\frac{1}{2}\left(\gamma_{\Phi}+\gamma_{n}\right)$. The instantaneous mode frequency as a function of the normalized time for the electrostatic potential (plain line) and the perturbed plasma density (dotted line) is shown in Figure 5.

As discussed in the previous section, the field-line averaged profiles $\langle\widehat{\Phi}\rangle_{\bar{\theta}}$ and $\langle\widehat{n}\rangle_{\bar{\theta}}$ depend implictly on the parameter $\bar{\theta}_{\max }$. Therefore one must ensure that the parameter $\bar{\theta}_{\max }$ is large enough so that the linear growth rate and the mode frequency become independent of its value. Figure 6 shows the average linear growth rate, $\gamma_{\ell}$, as a function of the parameter $\bar{\theta}_{\max }$. The parameters used in the simulations are: $\alpha_{b}=0, s_{b}=0.25, q=2.28, n_{0}=5 \times 10^{12} \mathrm{~cm}^{-3}$, $T_{e}=5 \mathrm{eV}, k_{\theta} \rho_{\mathrm{s} 0}=0.8, B_{0}=10^{4}$ Gauss, $\Delta \bar{\theta}=\pi / 128, L_{n}=4.0 \mathrm{~cm}, R_{0}=10^{2} \mathrm{~cm}, \epsilon_{\mathrm{t}}=0.2$, $\epsilon_{\mathrm{h}}=0.05$. For $\bar{\theta}_{\max }>5$, the linear growth rate reaches its asymptotic value. Note that in practice the parameter $\bar{\theta}_{\text {max }}$ must be increased as the global shear parameter, $s_{b}$, is decreased (the extent of the mode along the field line increases with decreasing global shear).

Figure 7 shows the average linear growth rate as a function of the helical parameter, $\epsilon_{\mathrm{h}}$. The toroidicity parameter is kept constant at $\epsilon_{\mathrm{t}}=0.2$. Other parameters are the same as in Figure 6. As mentioned in the beginning of this section, the case $\epsilon_{\mathrm{h}}=0$ corresponds to the tokamak parameterization (with circular magnetic surfaces). The linear growth rate increases until $\epsilon_{\mathrm{h}} \sim \epsilon_{\mathrm{t}}$. Note that each square corresponds to a different magnetic configuration; it takes about 55 seconds on a workstation to compute the MHD equilibrium and the linear growth rate. If one uses a global equilibroum code, one would have to recalculate the MHD equilibrium for the entire plasma volume for each data point in Figure 7: the use of the local equilibrium model represents an enormous reduction in the overall computational effort.

In order to understand the dependence of the linear growth rate on the helical parameter, one can compare the key attributes of the magnetic configuration for specific sets $\left(\epsilon_{\mathrm{t}}, \epsilon_{\mathrm{h}}\right)$; such key attributes are the normal and geodesic components of the magnetic curvature $\left(\kappa_{n}\right.$ and $\kappa_{g}$, respectively), the magnetic shear, $S$, and the normal torsion, $\tau_{n}$. Other quantities that characterize the equilibrium configuration do enter the drift wave stability calculations; however, the impact of these quantities are subdominant. The normal magnetic curvature and the magnetic shear are probably the most important quantities that affect drift wave (and ballooning) stability [5]. Figure 8 shows the normal curvature (plain line) and the local magnetic shear (dotted line) along the field line for the case of $\left(\epsilon_{\mathrm{t}}, \epsilon_{\mathrm{h}}\right)=(0.2,0.05)$. The ballooning parameter is $\alpha_{b}=0$ and the global shear parameter is $s_{b}=0.25$. For these parameters, the modes are nonzero in the range $|\bar{\theta}| \lesssim 5$ (See Figures 2 and 3). Figure 8 shows that the normal curvature is destabilizing (negative) in the outboard side of the torus. The magnetic shear is positive around $\bar{\theta}=0$ but becomes negative further away along the field line. The normal curvature (plain line) and the local magnetic shear (dotted line) along the field line for the case of $\left(\epsilon_{\mathrm{t}}, \epsilon_{\mathrm{h}}\right)=(0.2,0.2)$ is shown in Figure 9. The normal curvature has a more destabilizing influence on the drift modes as compared to Figure 8; this is one indication that the linear growth rate for the parameters of Figure 9 should be larger than for the case of Figure 8. However, one must also consider the impact of the local magnetic shear. Note that in the case of Figure 9 the bulk of the drift 
mode amplitude experiences a positive global shear away from the $\bar{\theta}=0$; therefore, we expect the linear growth rate to be larger for the case of Figure 9 as compared to the case of Figure 8. The detrimental influence of a large, positive local magnetic shear on drift wave stability in realistic 3D stellararator geometries has been noted by Nadeem and co-workers [8]; these authors also discuss the case of large, negative local magnetic shear which appears to have a stabilizing influence on the drift mode. This is in agreement with our observations, although our model equilibrium is far simpler than the fully 3D stellarator equilibrium used in the work of Nadeem et al.

Since the collisional parameter scales linearly with the electron collision time, and noting that $\tau_{e} \propto T_{e}^{3 / 2}$, we expect the linear growth rate to decrease sharply with increasing electron temperature. As the electron temperature increases, the electron mean free path, $\lambda_{e}$, increases; when $\lambda_{e} \gtrsim k_{\|}^{-1}$, the electron response becomes adiabatic. Figure 10 shows the linear growth rate normalized to the drift frequency, $\widetilde{\gamma} \equiv \gamma / \omega_{\star}$. As expected, the linear growth rate decreases with increasing electron temperature; however, since the drift frequency scales like $\sqrt{T_{e}}$, it is more meaningfull to normalize the linear growth rate as

$$
\frac{\gamma}{\gamma_{0}}=\frac{\widetilde{\gamma}}{\widetilde{\gamma}_{0}} \sqrt{\frac{T_{e 0}}{T_{e}}}
$$

where $\gamma_{0}$ denotes the linear growth rate evaluated at $T_{e}=T_{e 0}$. Figure 11 shows a plot of $\gamma / \gamma_{0}$ as a function of the electron temperature; the electron temperature of reference is $T_{e 0}=5 \mathrm{eV}$. The linear growth rate based on this simplified two-field model depends strongly on the electron temperature.

The dependence of the normalized linear growth rate on the global shear parameter, $s_{b}$, is shown in Figure 12. The toroidicity and helical parameters are $\epsilon_{\mathrm{t}}=0.2$ and $\epsilon_{\mathrm{h}}=0.1$, respectively. Figure 12 shows that there are two regimes: the low-shear regime, defined somewhat arbitrarily as $s_{b}<1$, and the high-shear regime, $s_{b}>1$. The dependence of the linear growth rate is approximately linear in these two regimes: $\gamma / \omega_{\star}=0.18-0.048 s_{b}$ in the low-shear regime; and $\gamma / \omega_{\star}=0.14-0.015\left(s_{b}-1\right)$ in the high-shear regime. The fact that the variation of the linear growth with $s_{b}$ is steeper in the low-shear regime is that, for small $s_{b}$, the global magnetic shear $(\langle S\rangle)$ is comparable to, or smaller than, the magnitude of the shear that varies in the magnetic

surface, $\widetilde{S} \equiv S-\langle S\rangle$. In the high-shear regime, the contribution of $\widetilde{S}$ becomes less important and the dependence of $\gamma$ depends mostly on the global shear $\langle S\rangle$.

\section{Conclusions}

Drift wave stability calculations in stellarator geometry usually requires the use of sophisticated, computationally-intensive 3D MHD equilibrium codes. However, as far as linear stability is concerned, the general approach is to study the local drift wave stability using the ballooning representation, one magnetic surface at a time. Hegna's local equilibrium model [3] is specific to a particular magnetic surface and it is computationally very efficient.

The local equilibrium model has been used to study the drift wave stability properties of a two-field resistive model valid in the low-temperature, high-density edge plasmas of tokamaks and stellarators. It has been shown that in the case of a stellarator with a helical axis a large, positive magnetic shear can increase the linear growth rate, whereas a large, negative magnetic shear has a stabilizing influence on the drift modes. 


\section{A Derivation of the Resistive Drift Wave Model}

In the cold ion limit, the ion momentum equation reads

$$
m_{i} n\left(\frac{\partial}{\partial t}+\mathbf{V}_{i} \cdot \boldsymbol{\nabla}\right) \mathbf{V}_{i}=e n\left(\mathbf{E}+\frac{\mathbf{V}_{i} \times \mathbf{B}}{c}\right)-\mathbf{R}_{e i}
$$

where $\mathbf{R}_{e i}=e n\left(\mathbf{J}_{\|} / \sigma_{\|}+\mathbf{J}_{\perp} / \sigma_{\perp}\right)-0.71 n \nabla_{\|} T_{e}-\frac{3}{2} n \widehat{\mathbf{b}} \times \nabla_{\perp} T_{e} /\left(\omega_{c e} \tau_{e}\right)$ is the momentum transfer due to collisions; $\sigma_{\|} \simeq 2 \sigma_{\perp}, \sigma_{\perp}=e^{2} n \tau_{e} / m_{e}$ are the parallel and perpendicular electron conductivities, respectively; $\tau_{e}=\left(3 \sqrt{m_{e}} T_{e}^{3 / 2}\right) /\left(4 \sqrt{2 \pi} n \lambda e^{4}\right)$ is the electron collision time. The electron momentum equation (for massless electrons) is

$$
\nabla p_{e}+e n\left(\mathbf{E}+\frac{\mathbf{V}_{e} \times \mathbf{B}}{c}\right)=\mathbf{R}_{e i} .
$$

Operating with $\mathbf{B} \times$ on Eqs. $(35,36)$ we obtain (in the low-frequency regime of $\omega / \omega_{c i} \ll 1$ )

$$
\mathbf{V}_{i \perp}=\mathbf{V}_{E}+\mathbf{V}_{p i}+\mathbf{V}_{c}
$$

and

$$
\mathbf{V}_{e \perp}=\mathbf{V}_{E}+\mathbf{V}_{\star e}+\mathbf{V}_{c}
$$

respectively. Here

$$
\begin{aligned}
\mathbf{V}_{E} & =\frac{c}{B^{2}} \mathbf{B} \times \nabla \Phi, \\
\mathbf{V}_{p i} & =\omega_{c i}^{-1} \widehat{\mathbf{b}} \times\left(\frac{\partial}{\partial t}+\mathbf{V}_{E} \cdot \nabla\right) \mathbf{V}_{E}, \\
\mathbf{V}_{c} & =\frac{c}{e n B^{2}} \mathbf{B} \times \mathbf{R}_{e i}, \\
\mathbf{V}_{\star e} & =-\frac{c}{e n B^{2}} \mathbf{B} \times \nabla p_{e},
\end{aligned}
$$

are the $\mathbf{E} \times \mathbf{B}$ drift velocity (in the low- $\beta$ approximation of $\mathbf{E} \simeq-\boldsymbol{\nabla} \Phi$ ), the ion polarization drift velocity, the collisional drift velocity and the electron diamagnetic drift velocity, respectively. For low-frequency, long-wavelength modes $\left(k_{\perp}^{2} \lambda_{D}^{2} \ll 1\right.$ ) (where $k_{\perp}$ is the magnitude of the perpendicular wavevector and $\lambda_{D}$ is the Debye length), the plasma is quasineutral

$$
\nabla \cdot \mathbf{J}=0
$$

or

$$
\boldsymbol{\nabla} \cdot \mathbf{J}_{\perp}=-\mathbf{B} \cdot \boldsymbol{\nabla}\left(\frac{J_{\|}}{B}\right),
$$

where the perpendicular current density is obtained from Eqs. $(37,38)$

$$
\mathbf{J}_{\perp}=e n\left(\mathbf{V}_{p i}-\mathbf{V}_{\star e}\right)
$$


and the parallel current density, $J_{\|}$, is determined from the scalar product of Eq.(36) with $\widehat{\mathbf{b}} \equiv \mathbf{B} / B$

$$
J_{\|}=\frac{\sigma_{\|}}{e n}\left(\nabla_{\|} p_{e}+0.71 n \nabla_{\|} T_{e}-e n \nabla_{\|} \Phi\right)
$$

Combining Eqs.(40-42) and linearizing, we obtain the quasineutrality equation (for $V_{i \|} \ll V_{e \|}$ ) as

$$
\frac{n_{0} c}{B \omega_{c i}} \frac{\partial}{\partial t} \nabla_{\perp}^{2} \Phi=2 n_{0} \frac{\nabla B}{B} \cdot \mathbf{V}_{\star e}+\mathbf{B} \cdot \nabla\left[\frac{\sigma_{\|}}{e n_{0} B}\left(\nabla_{\|} p_{e}+0.71 n \nabla_{\|} T_{e}-e n \nabla_{\|} \Phi\right)\right]
$$

where we made use of the (low- $\beta$ ) relation of

$$
\boldsymbol{\nabla} \cdot \frac{\mathbf{B} \times \nabla f}{B^{2}} \simeq-2 \frac{\nabla B}{B} \cdot \frac{\mathbf{B} \times \nabla f}{B^{2}}
$$

for any fluctuating quantity $f$. Using the definitions (39) in the electron continuity equation

$$
\frac{\partial n}{\partial t}=-\boldsymbol{\nabla} \cdot\left(n \mathbf{V}_{E}\right)-\boldsymbol{\nabla} \cdot\left(n \mathbf{V}_{\star e}\right)-\boldsymbol{\nabla} \cdot\left(n \mathbf{V}_{c}\right)
$$

and noting that $V_{c} \sim V_{E} /\left(\omega_{c e} \tau_{e}\right) \ll V_{E} \sim V_{\star e}$, one gets

$$
\frac{\partial n}{\partial t}=\left(2 \frac{\boldsymbol{\nabla} B}{B}+\frac{\boldsymbol{\nabla} \rho}{L_{n}}\right) \cdot\left(n_{0} \mathbf{V}_{E}\right)+2 \frac{\boldsymbol{\nabla} B}{B} \cdot\left(n_{0} \mathbf{V}_{E}\right)+\frac{1}{e} \mathbf{B} \cdot \boldsymbol{\nabla}\left(\frac{J_{\|}}{B}\right) .
$$

Here $\rho=\rho(\psi)$ is a radial coordinate $(\mathbf{B} \cdot \nabla \rho=0)$. In drift wave units $\left[\bar{t}=\omega_{\star} t, \omega_{\star}=c_{s} / L_{n}\right.$ is the drift frequency, $c_{s}=\sqrt{T_{e} / m_{i}}$ is the sound speed, $\left.(\widetilde{n}, \widetilde{\Phi})=\left(\delta n / n_{0}, e \Phi / T_{e}\right)\right]$, Eqs. $(43,46)$ become (neglecting electron temperature fluctuations)

$$
\frac{1}{\bar{B}^{2}} \frac{\partial \bar{\omega}}{\partial \bar{t}}=2 \xi \bar{B} L_{n} \nabla_{\|}\left(\frac{L_{n}}{\bar{B}} \nabla_{\|} \widetilde{h}\right)-2 \mathbf{Q} \cdot \frac{\widehat{\mathbf{b}} \times \rho_{\mathrm{s} 0} \nabla \widetilde{n}}{\bar{B}}
$$

and

$$
\frac{\partial \widetilde{n}}{\partial \bar{t}}=(2 \mathbf{Q}+\nabla \rho) \cdot \frac{\widehat{\mathbf{b}} \times \rho_{\mathrm{s} 0} \nabla \widetilde{\Phi}}{\bar{B}}-2 \mathbf{Q} \cdot \frac{\widehat{\mathbf{b}} \times \rho_{\mathrm{s} 0} \boldsymbol{\nabla} \widetilde{n}}{\bar{B}}+2 \xi \bar{B} L_{n} \nabla_{\|}\left(\frac{L_{n}}{\bar{B}} \nabla_{\|} \widetilde{h}\right)
$$

where $\bar{\omega} \equiv \rho_{\mathrm{s} 0}^{2} \nabla_{\perp}^{2} \widetilde{\Phi}$ and $\xi \equiv \omega_{\star} \tau_{e}\left(m_{i} / m_{e}\right) \gg 1$ is termed the collisional parameter; $\mathbf{Q} \equiv$ $L_{n} \nabla B / B$ is related to the curvature of the magnetic field and $\rho_{\mathrm{s} 0}=\sqrt{T_{e} / m_{i}} /\left(e B_{0} / m_{i} c\right)$; $\bar{B} \equiv B / B_{0}$ where $B_{0}$ is a magnetic field of reference (see main text); finally $\widetilde{h}=\widetilde{n}-\widetilde{\Phi}$ is the nonadiabatic response of the electrons. 


\section{B Remarks on the Local Magnetic Shear}

Using the definitions of the unit parallel vector, $\widehat{\mathbf{b}}=\mathbf{B} / B$, and the unit normal vector, $\widehat{\mathbf{n}}=$ $\boldsymbol{\nabla} \psi / \sqrt{g^{\psi \psi}}$, we can write the binormal vector as

$$
\widehat{\mathrm{g}}=f(\mathbf{B} \times \nabla \psi),
$$

where $f=\left(B \sqrt{g^{\psi \psi}}\right)^{-1}$. Using Eq.(49) in the definition of the local magnetic shear we obtain

$$
\begin{aligned}
S & =\widehat{\mathbf{g}} \cdot \boldsymbol{\nabla} \times \widehat{\mathbf{g}}=\widehat{\mathbf{g}} \cdot[\boldsymbol{\nabla} f \times(\mathbf{B} \times \boldsymbol{\nabla} \psi)+\boldsymbol{\nabla} \times(\mathbf{B} \times \boldsymbol{\nabla} \psi)], \\
& =f(\boldsymbol{\nabla} g^{\psi \psi} \cdot \underbrace{\nabla \alpha \times \widehat{\mathbf{g}}}_{\mathbf{1}}-\boldsymbol{\nabla} g^{\alpha \psi} \underbrace{\boldsymbol{\nabla} \psi \times \widehat{\mathbf{g}}}_{\mathbf{2}}),
\end{aligned}
$$

where we have used the Clebsch for the magnetic field, $\mathbf{B}=\boldsymbol{\nabla} \alpha \times \boldsymbol{\nabla} \psi$. Terms $\mathbf{1}$ and $\mathbf{2}$ in Eq.(50) can be written as

$$
\boldsymbol{\nabla} \alpha \times \widehat{\mathbf{g}}=g \boldsymbol{\nabla} \alpha \times(\mathbf{B} \times \boldsymbol{\nabla} \psi)=f g^{\alpha \psi} \mathbf{B}
$$

and

$$
\boldsymbol{\nabla} \psi \times \widehat{\mathrm{g}}=f \boldsymbol{\nabla} \psi \times(\mathbf{B} \times \nabla \psi)=\frac{\sqrt{g^{\psi \psi}}}{B} \mathbf{B},
$$

respectively. Combining Eqs.(50-52), one can write

$$
\begin{aligned}
S & =f\left(f g^{\alpha \psi} \mathbf{B} \cdot \boldsymbol{\nabla} g^{\psi \psi}-\frac{\sqrt{g^{\psi \psi}}}{B} \mathbf{B} \cdot \boldsymbol{\nabla} g^{\alpha \psi}\right), \\
& =-\frac{|\boldsymbol{\nabla} \psi|^{2}}{B^{2}} \mathbf{B} \cdot \boldsymbol{\nabla}\left(\frac{g^{\alpha \psi}}{g^{\psi \psi}}\right), \\
& =\frac{|\boldsymbol{\nabla} \psi|^{2}}{B^{2}} \mathbf{B} \cdot \boldsymbol{\nabla}\left(D+\zeta \frac{d \iota}{d \psi}\right),
\end{aligned}
$$

where

$$
D \equiv \frac{\iota \nabla \zeta \cdot \nabla \psi-\nabla \theta \cdot \nabla \psi}{\nabla \psi}
$$




\section{Conventions}

There is some freedom in defining key dimensionless parameters. To make connection with previous work, we consider the expression for the local magnetic shear for a tokamak with circular magnetic surfaces and make a one-to-one correspondance with the three-dimensional local model. For a tokamak plasma with circular magnetic surfaces, the magnetic surfaces are parameterized according to

$$
\begin{aligned}
R & =R_{0}+r \cos \theta_{0}, \\
\phi & =-\zeta, \\
Z & =r \sin \theta_{0} .
\end{aligned}
$$

In the large-aspect-ratio limit, the geometrical poloidal angle, $\theta_{0}$, is approximately equal to the magnetic poloidal angle, $\theta$. Using Eq.(55) we can easily calculate the metrics $g_{\theta \theta}=r^{2}, g_{\zeta \zeta}=R^{2}$ and $g_{\theta \zeta}=0$ (since the basis vectors $\mathbf{e}_{\theta}$ and $\mathbf{e}_{\zeta}$ are orthogonal in this limit). The Jacobian is given by $\mathcal{J}=C R^{2}$ where the constant $C$ is determined from the normalization condition $\int_{0}^{2 \pi} d \zeta \int_{0}^{2 \pi} d \theta \mathcal{J}=V^{\prime}$, which yields

$$
\mathcal{J} \simeq \frac{V^{\prime}}{4 \pi^{2} R_{0}^{2}}
$$

where $V^{\prime}=d V / d \psi$. The toroidal flux function is given by $\psi \simeq B_{0} r^{2} / 2$ where $B_{0}$ is the magnetic field strength on the magnetic axis. Using the parameterization (55) we obtain

$$
\frac{B^{2}}{g^{\psi \psi}}=\frac{\iota^{2} g_{\theta \theta}+2 \iota g_{\theta \zeta}+g_{\zeta \zeta}}{g_{\theta \theta} g_{\zeta \zeta}-g_{\theta \zeta}^{2}}=\frac{R^{2}+\iota^{2} r^{2}}{r^{2} R^{2}} \approx \frac{1}{r^{2}} \text {. }
$$

To calculate the local magnetic shear we must determine the expression for $\lambda$ (which is related to the parallel current flowing in the magnetic surface). Using $\mathbf{B} \cdot \boldsymbol{\nabla} \lambda=2|\boldsymbol{\nabla} \psi| \kappa_{g} / B$ and noting that the geodesic magnetic surface is $\kappa_{g} \simeq \sin \theta / R_{0}$,

$$
\frac{d \lambda}{d \theta} \simeq \frac{q r}{2 \pi^{2} R_{0}} V^{\prime} \sin \theta
$$

which can be integrated to give

$$
\lambda \simeq-\frac{q r}{2 \pi^{2} R_{0}} V^{\prime} \cos \theta
$$

To lowest order the flux surface averaged quantity $\langle\sigma\rangle$ is given by the global shear as

$$
\frac{4 \pi}{c}\langle\sigma\rangle\left\langle\frac{B^{2}}{g^{\psi \psi}}\right\rangle \simeq \frac{4 \pi^{2}}{V^{\prime}} \frac{d \iota}{d \psi} .
$$

The plasma volume enclosed within $r$ is $V=2 \pi^{2} r^{2} R_{0}$. Using $d \iota / d \psi=-q^{-2} d q / d r(d \psi / d r)^{-1}$ and $V^{\prime}=d V / d \psi=4 \pi^{2} r R_{0}(d \psi / d r)^{-1}$ we obtain from Eq.(59)

$$
\langle\sigma\rangle \simeq-\frac{c}{4 \pi} \frac{1}{q R_{0}} \widehat{s}
$$


where $\widehat{s}=r(d q / d r) / q$ is the usual (tokamak) definition of the global shear parameter. Using the definition of $\widehat{\sigma}=c \lambda d p / d \psi$ and Eqs. $(58,60)$ in the expression for the local magnetic shear, we obtain

$$
\left(-q R_{0}\right) S \simeq \widehat{s}+\frac{2}{\pi} r q^{2} \frac{d p}{d \psi} V^{\prime} \cos \theta .
$$

Defining the ballooning parameter

$$
\alpha_{B} \equiv-\frac{2}{\pi^{2} \iota^{2}} \frac{V^{\prime}}{\left\langle\frac{B^{2}}{g^{\psi \psi}}\right\rangle^{1 / 2}} \frac{d p}{d \psi},
$$

and the global shear parameter

$$
s_{B} \equiv-\frac{4 \pi^{2} R_{0}}{V^{\prime}} \frac{\frac{1}{\iota} \frac{d \iota}{d \psi}}{\left\langle\frac{B^{2}}{g^{\psi \psi}}\right\rangle},
$$

one can write Eq.(61) in the form of $\left(-q R_{0}\right) S=s_{B}-\alpha_{B} \cos \theta$. 
Acknowledgments This research was supported by Contract No. DE-AC02-CH0-3073 and the Scientific Discovery through Advanced Computing (SciDAC) initiative (U.S. Department of Energy). 


\section{References}

[1] W. Horton, Rev. Mod. Phys. 71, 735 (1999).

[2] J.W. Connor, R.J. Hastie and J.B. Taylor, Proc. Roy. Soc. London A 365, 1 (1979).

[3] C.C. Hegna, Phys. Plasmas 7(10), 3921 (2000).

[4] R. Courant, K. Friedrichs and H. Lewy, Mathematische Annalen 100, 32 (1928).

[5] J.L.V. Lewandowski, Drift wave models for three-dimensional plasmas (Ph.D. Thesis, the Australian National University, 1997).

[6] D.W. Peaceman and H.H. Rachford, J. Soc. Indust. Applied Mathematics 3(1), 28 (1955).

[7] J.L.V. Lewandowski, Phys. Plasmas 7(8), 3360 (2000).

[8] M. Nadeem, T. Rafiq and M. Persson, Phys. Plasmas 8(10), 4375 (2001). 
Figure 1 Schematic representation of the cross section $\zeta=0$ (see main text for definition of the average minor radius, $\bar{a}$ ).

Figure 2 Real (plain line) and imaginary (dotted line) parts of the mode amplitude for the normalized electrostatic potential at $\omega_{\star} t=10$.

Figure 3 Real (plain line) and imaginary (dotted line) parts of the mode amplitude for the normalized perturbed plasma density at $\omega_{\star} t=10$.

Figure 4 Instantaneous linear growth rates for the electrostatic potential (plain line) and the perturbed plasma density (dotted line) as a function of the normalized time $\omega_{\star} t$.

Figure 5 Instantaneous mode frequency for the electrostatic potential (plain line) and the perturbed plasma density (dotted line) as a function of the normalized time $\omega_{\star} t$.

Figure 6 Saturated linear growth rate as a function of the parameter $\bar{\theta}_{\max }$.

Figure 7 Saturated linear growth rate as a function of the helical parameter $\epsilon_{\mathrm{h}}$; the toroidicity parameter is kept fixed at $\epsilon_{\mathrm{t}}=0.2$.

Figure 8 Normal magnetic curvature (plain line) and local magnetic shear (dotted line) along the magnetic field line for the parameters of $\left(\epsilon_{\mathrm{t}}, \epsilon_{\mathrm{h}}\right)=(0.2,0.05)$.

Figure 9 Normal magnetic curvature (plain line) and local magnetic shear (dotted line) along the magnetic field line for the parameters of $\left(\epsilon_{\mathrm{t}}, \epsilon_{\mathrm{h}}\right)=(0.2,0.2)$.

Figure 10 Linear growth rate, normalized to the drift frequency, as a function of the electron temperature.

Figure 11 Linear growth rate, normalized to its value at $T_{e}=5 \mathrm{eV}$, as a function of the electron temperature.

Figure 12 Linear growth rate normalized to the drift frequency as a function of the global shear parameter $s_{b}$. 
FIG.1 Lewandowski

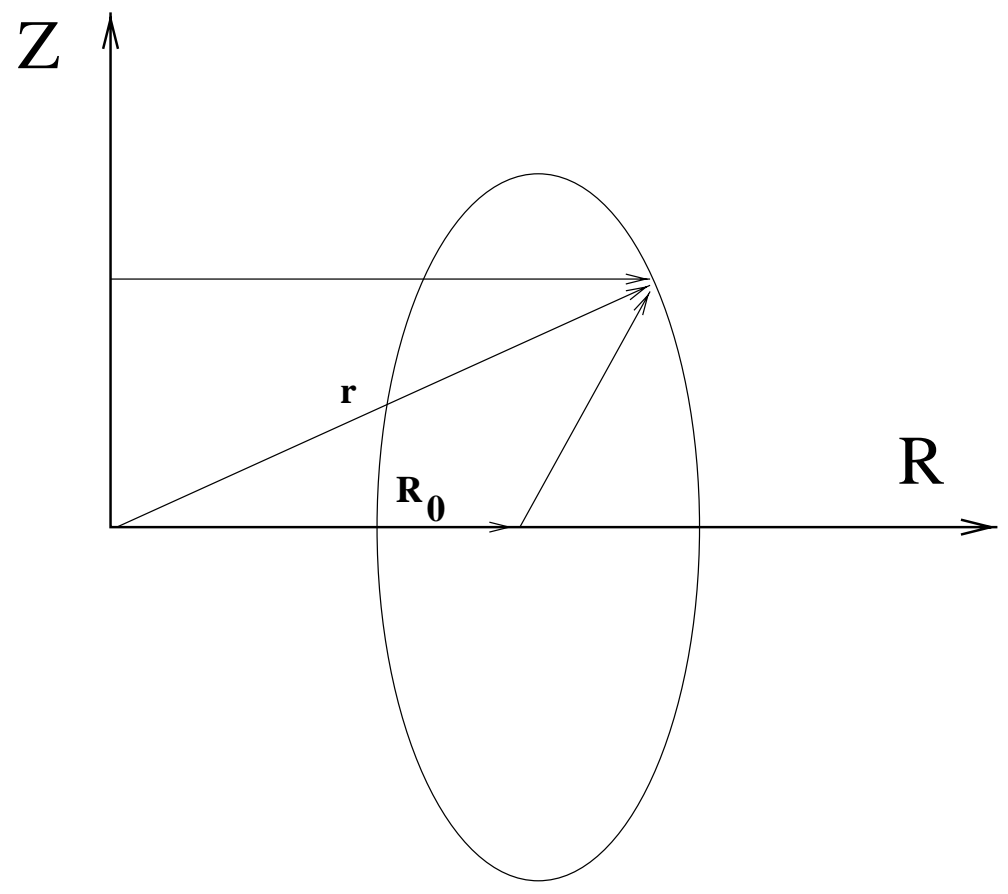




\section{FIG.2 Lewandowski}

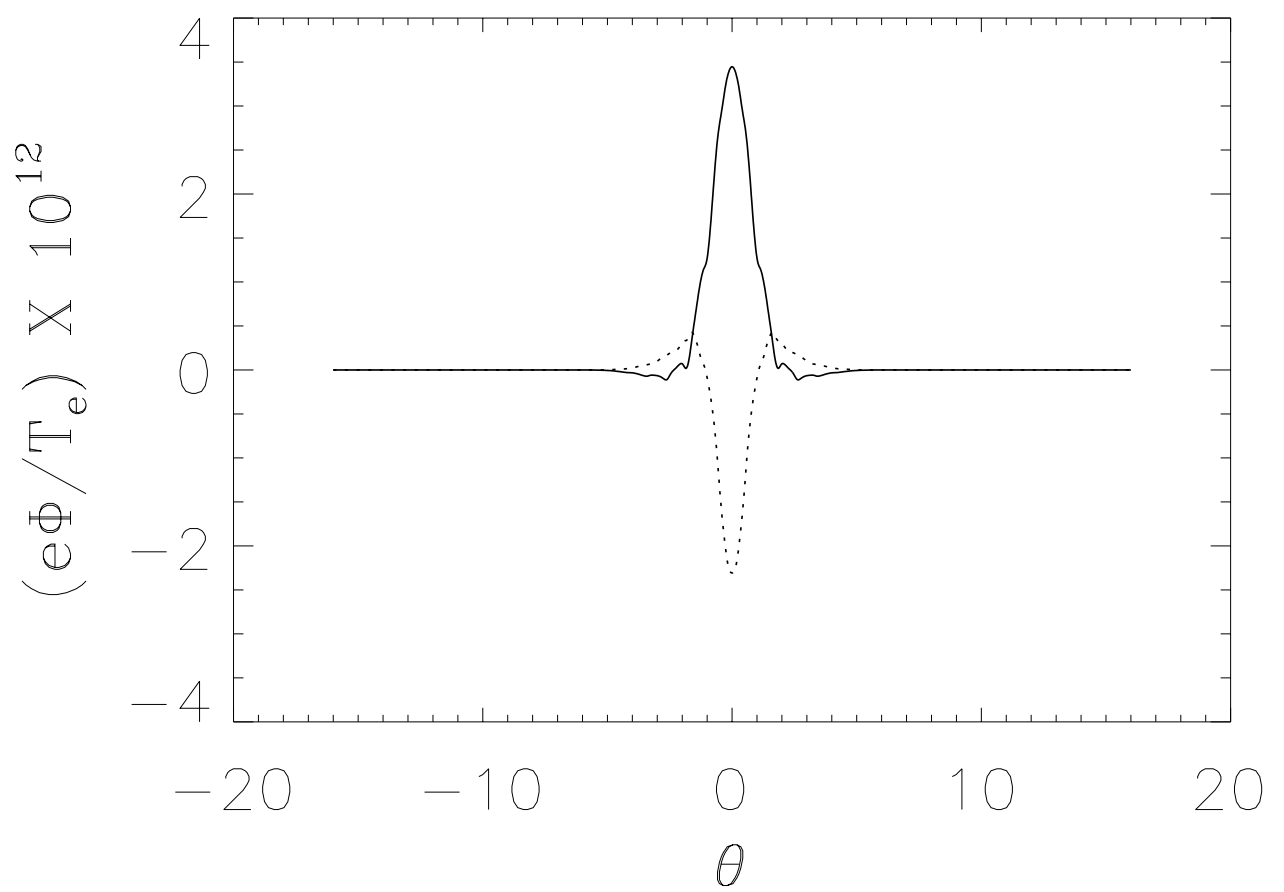




\section{FIG.3 Lewandowski}

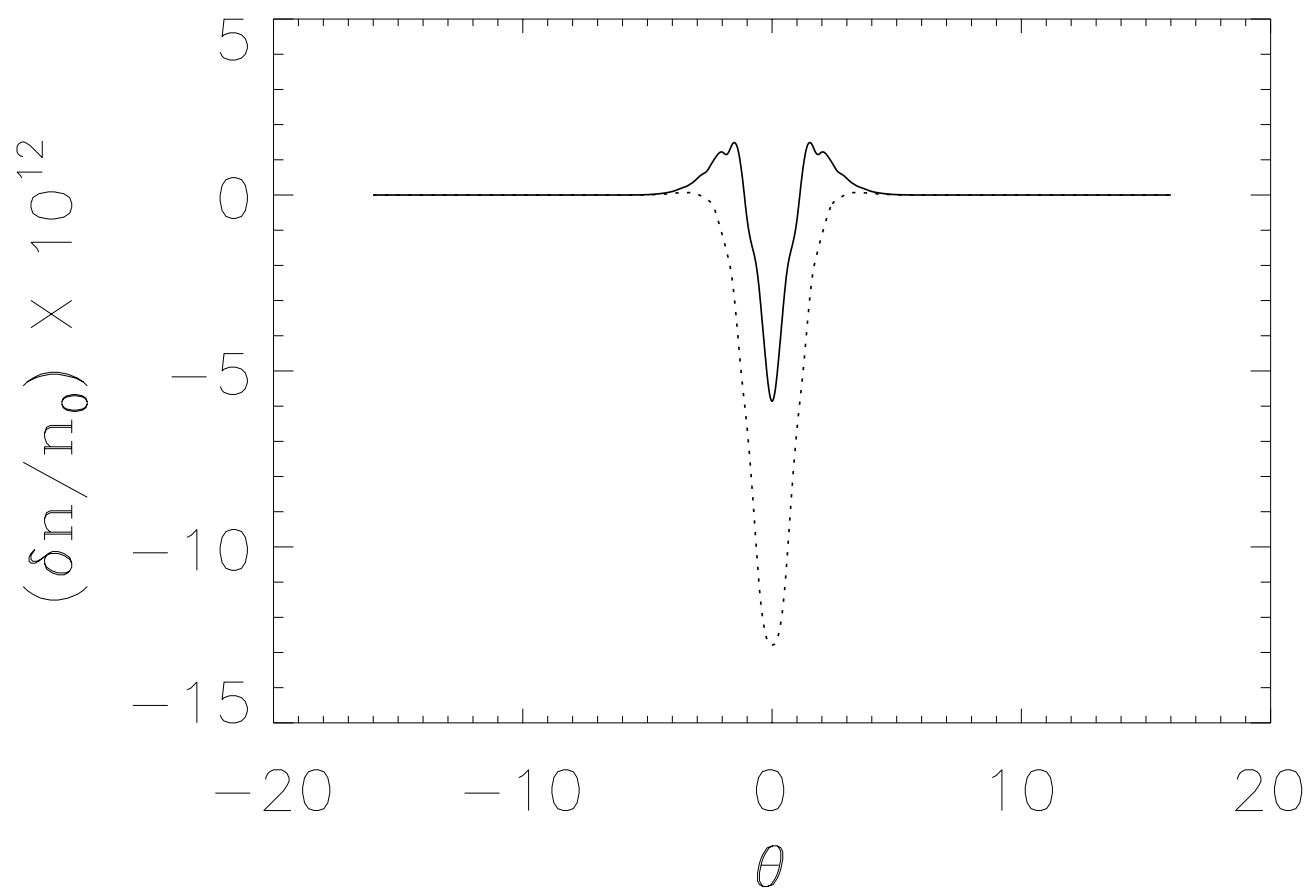




\section{FIG.4 Lewandowski}

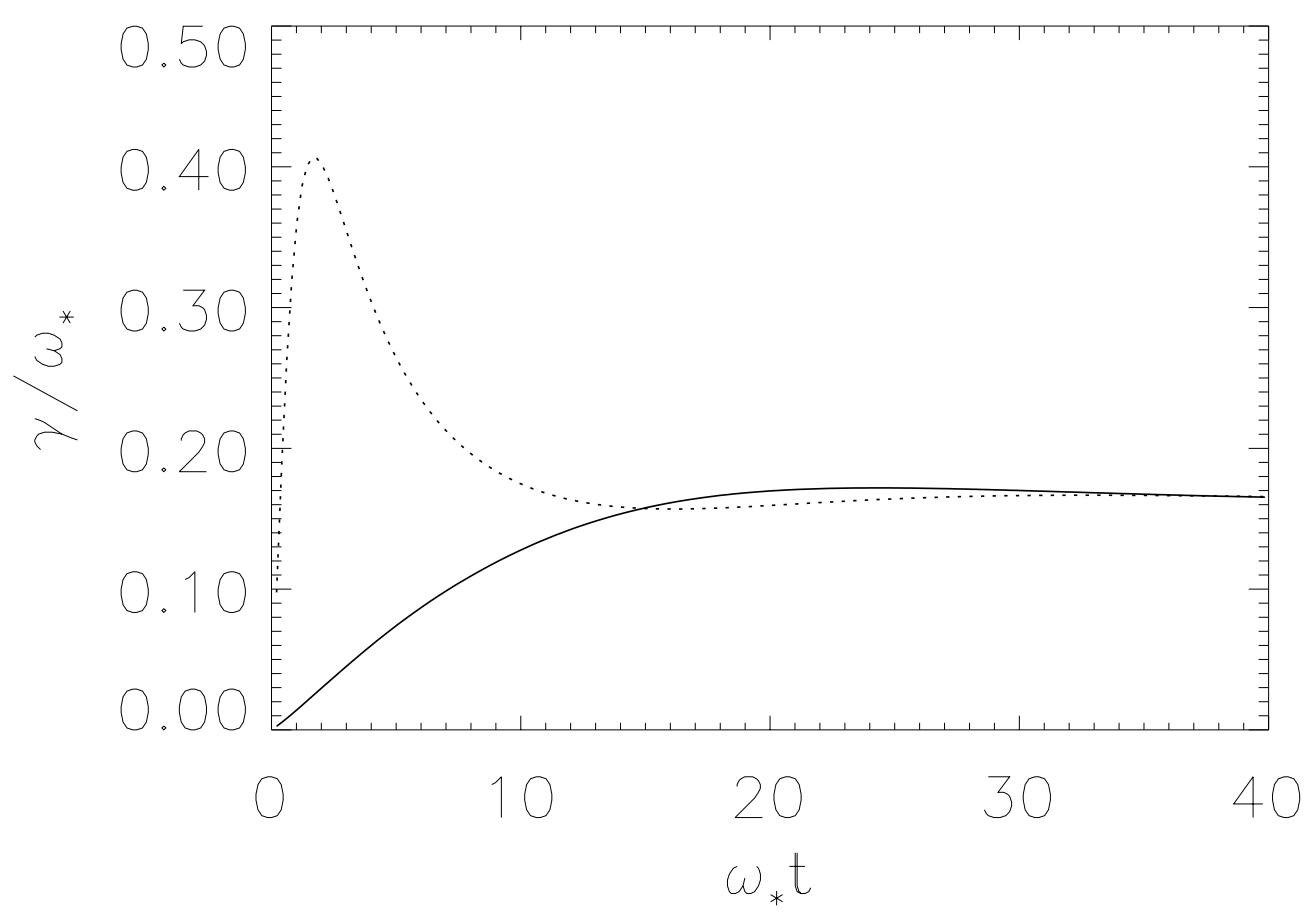




\section{FIG.5 Lewandowski}

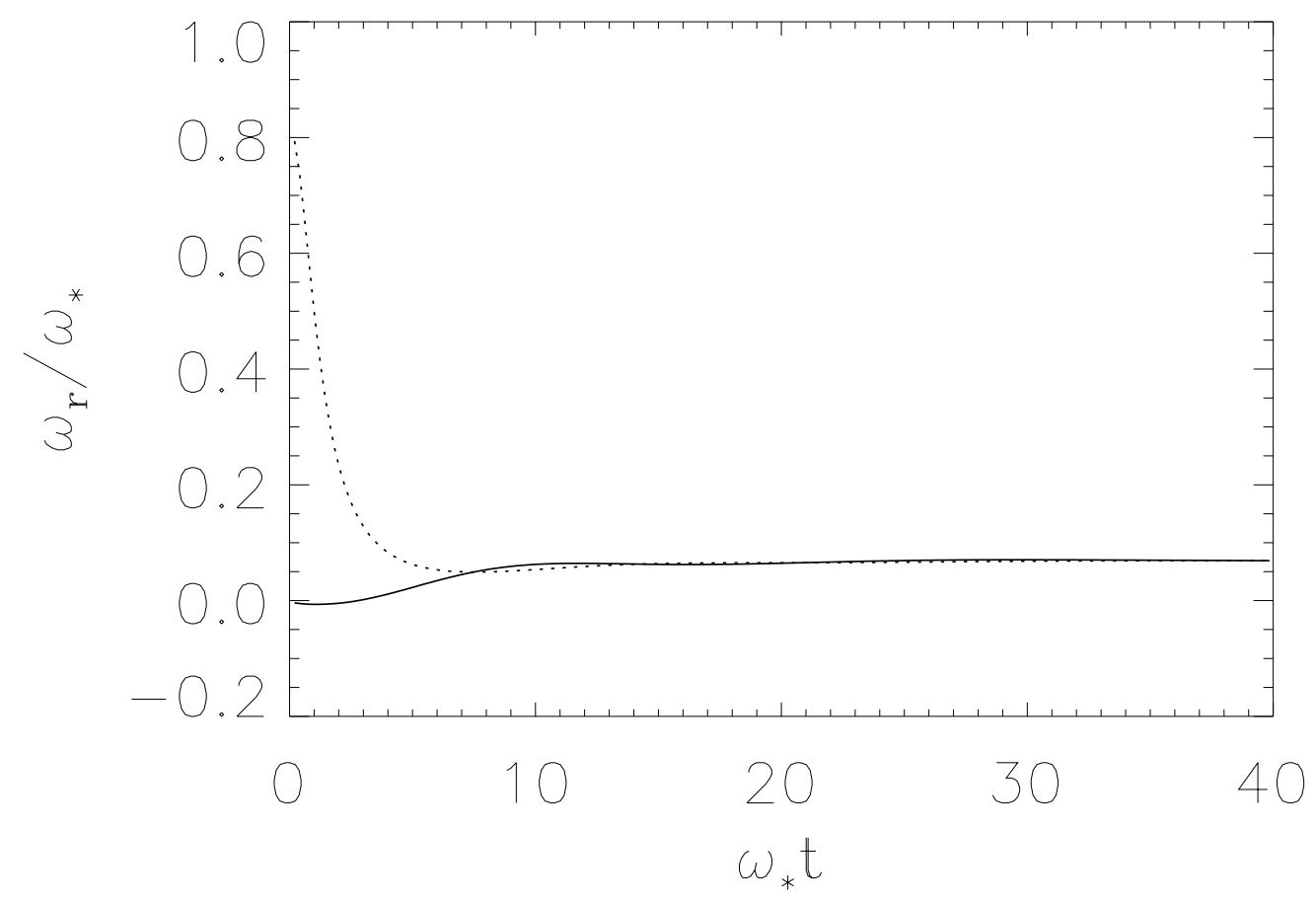




\section{FIG.6 Lewandowski}

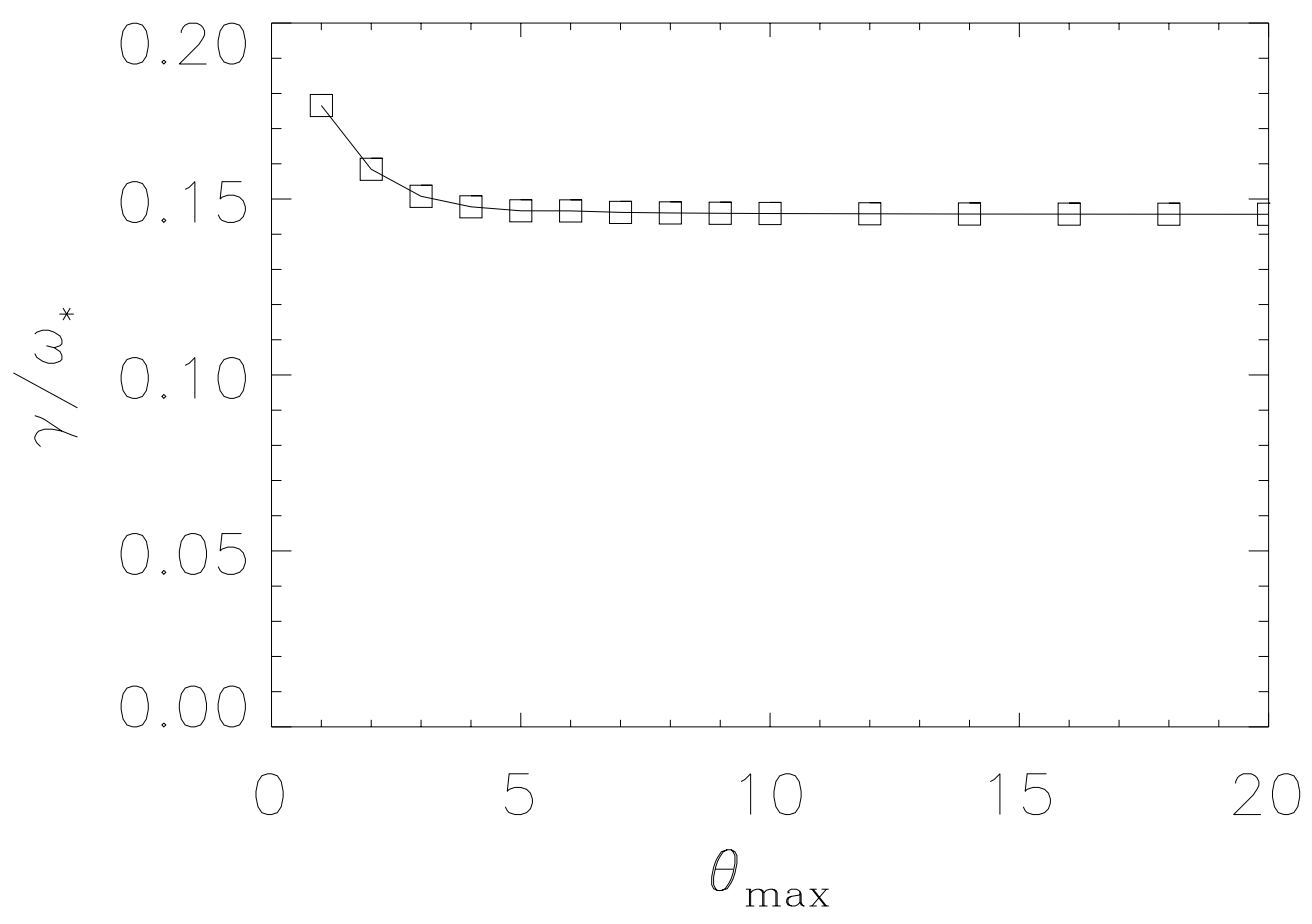




\section{FIG.7 Lewandowski}

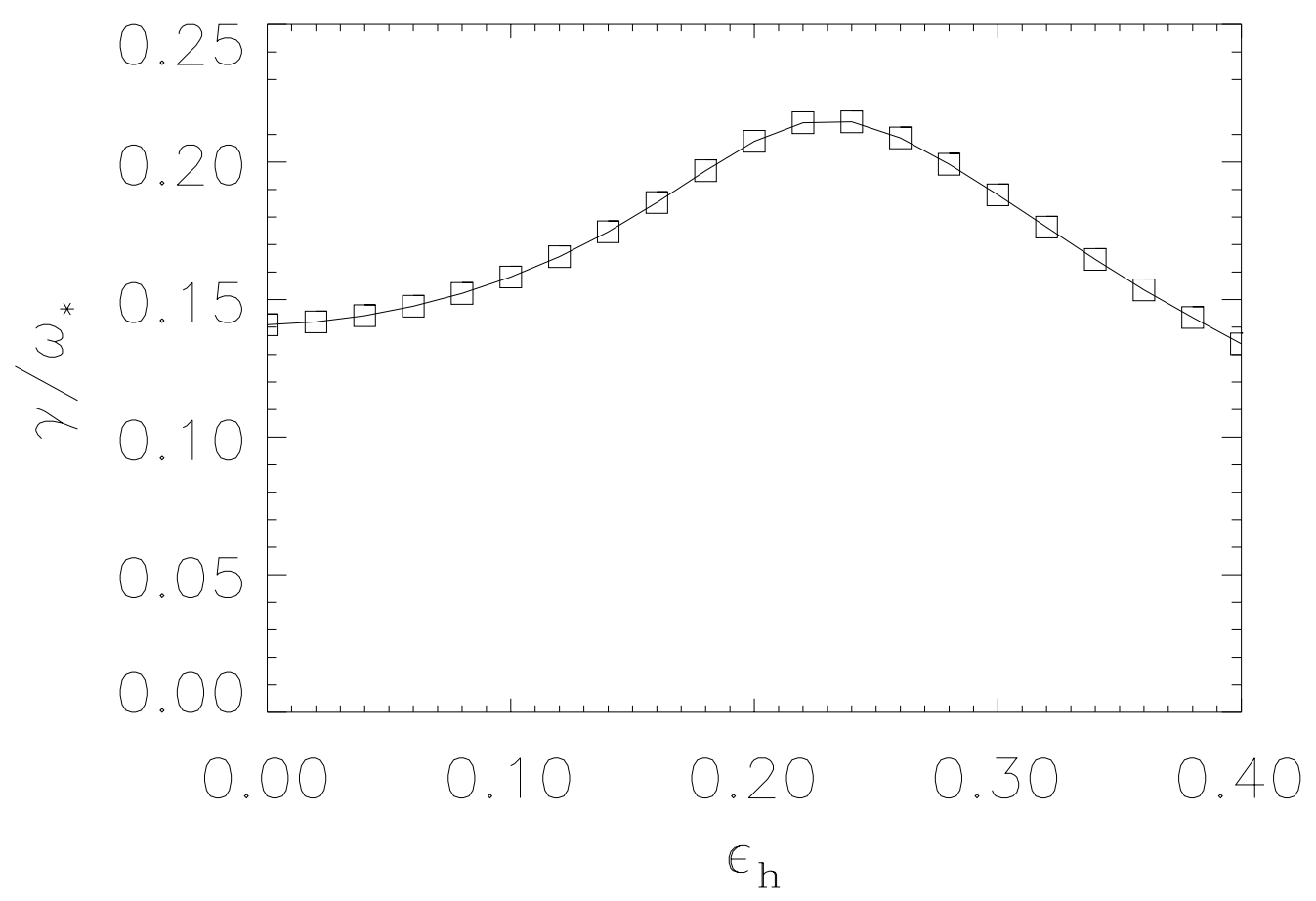




\section{FIG.8 Lewandowski}

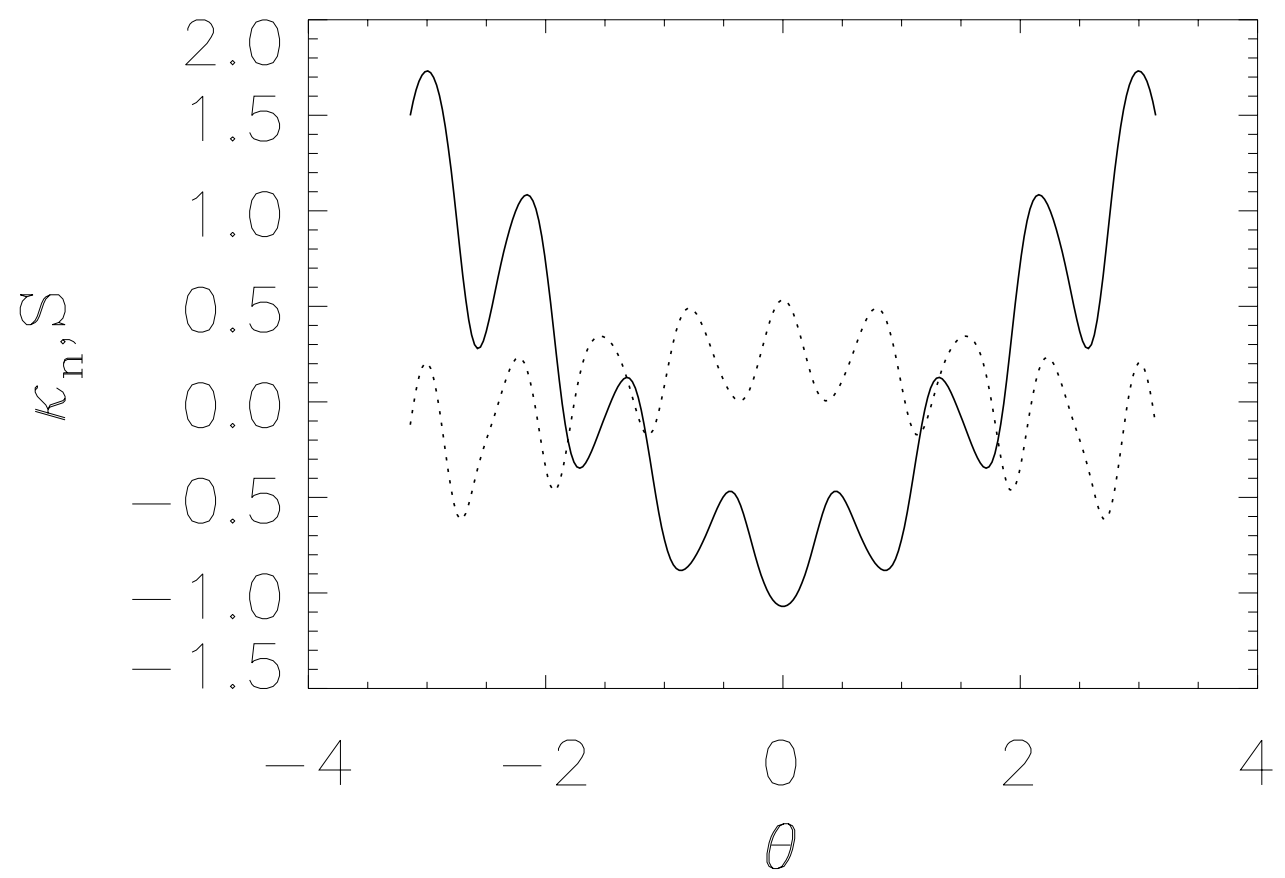




\section{FIG.9 Lewandowski}

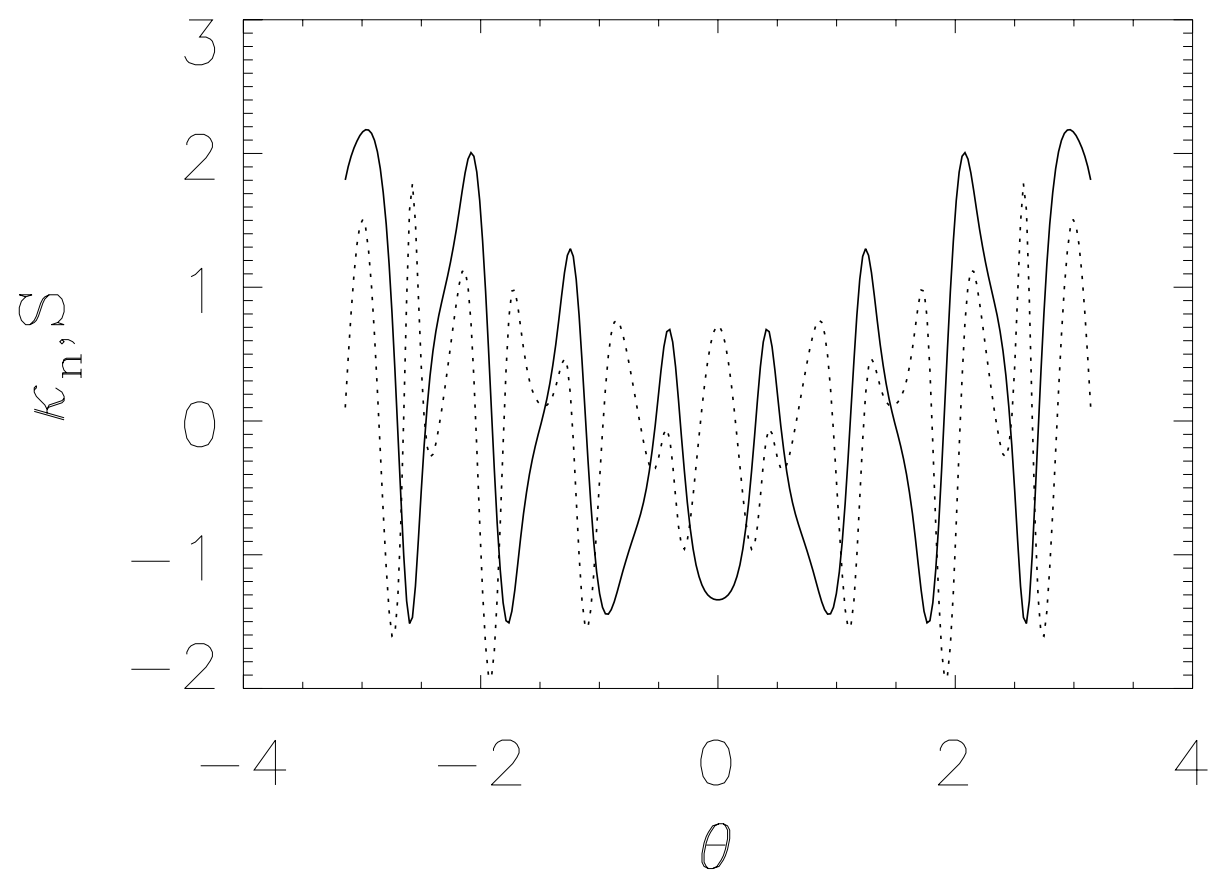


FIG.10 Lewandowski

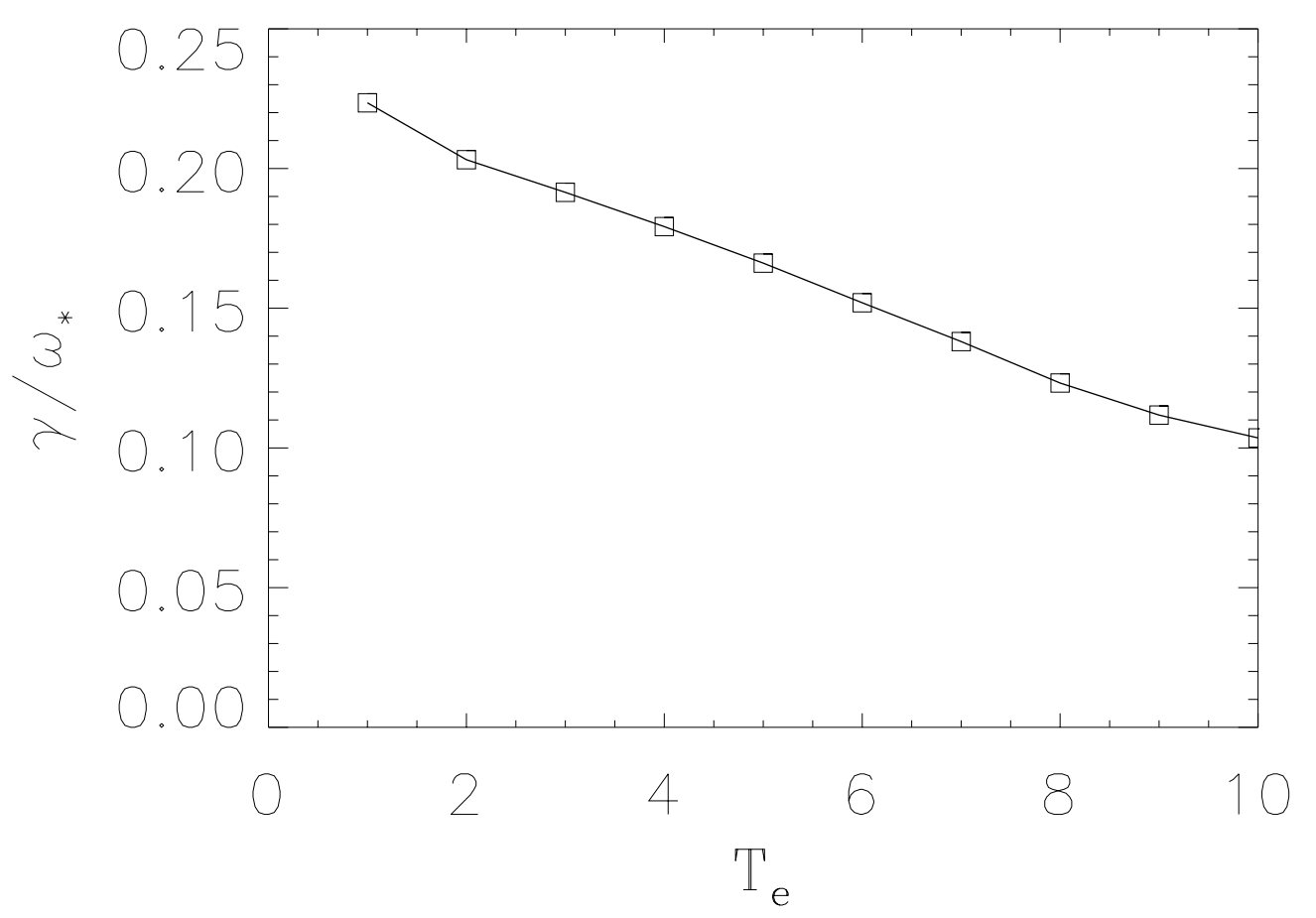




\section{FIG.11 Lewandowski}

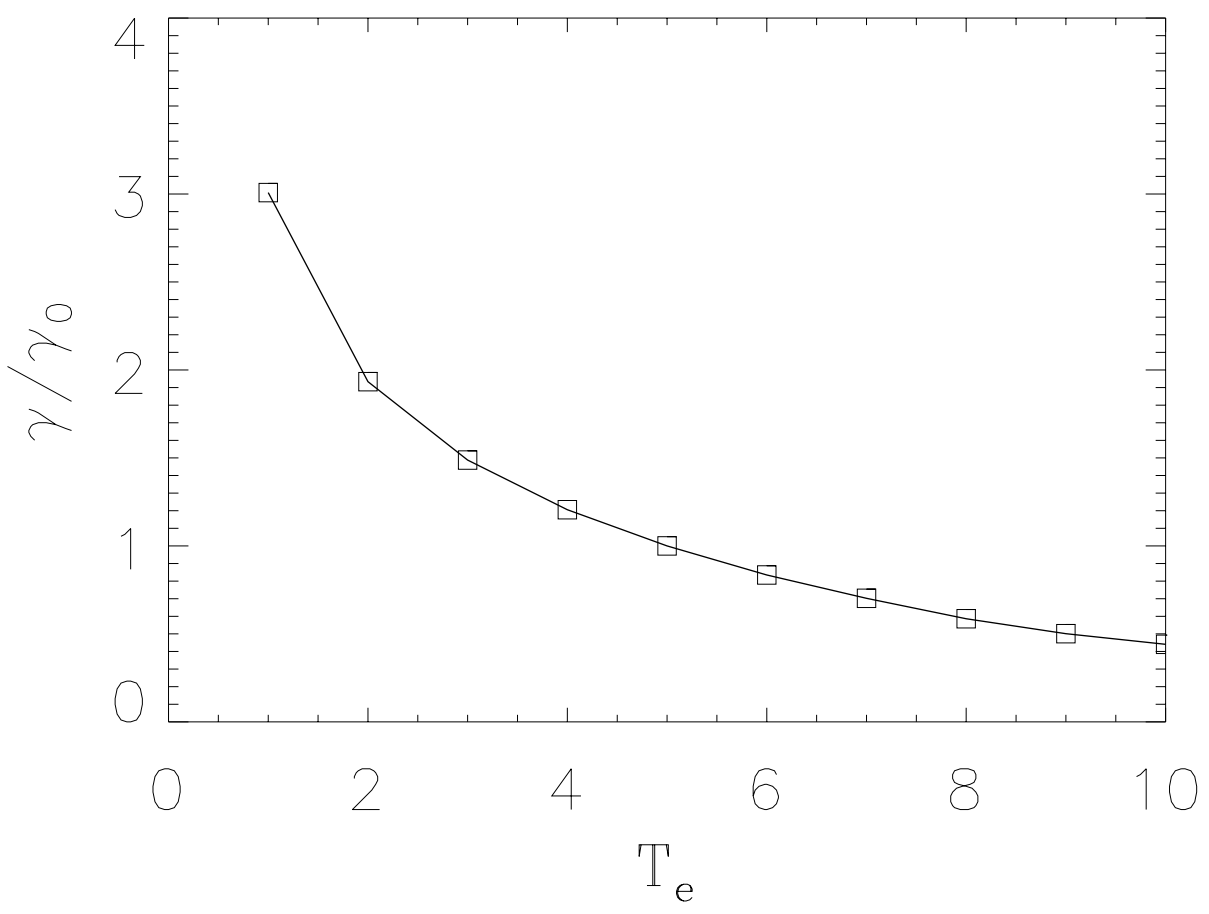




\section{FIG.121 Lewandowski}

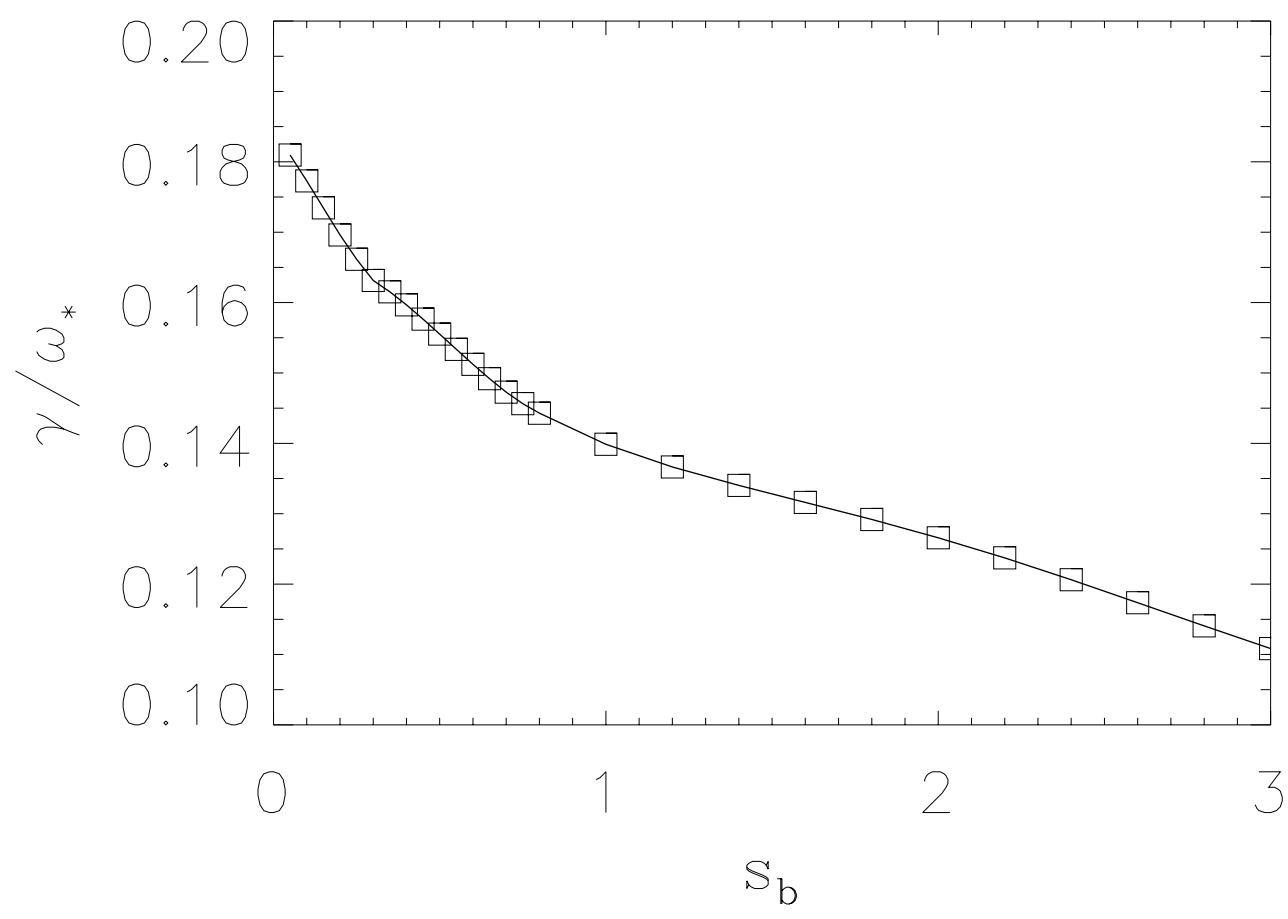




\section{External Distribution}

Plasma Research Laboratory, Australian National University, Australia

Professor I.R. Jones, Flinders University, Australia

Professor João Canalle, Instituto de Fisica DEQ/IF - UERJ, Brazil

Mr. Gerson O. Ludwig, Instituto Nacional de Pesquisas, Brazil

Dr. P.H. Sakanaka, Instituto Fisica, Brazil

The Librarian, Culham Laboratory, England

Mrs. S.A. Hutchinson, JET Library, England

Professor M.N. Bussac, Ecole Polytechnique, France

Librarian, Max-Planck-Institut für Plasmaphysik, Germany

Jolan Moldvai, Reports Library, Hungarian Academy of Sciences, Central Research Institute for Physics, Hungary

Dr. P. Kaw, Institute for Plasma Research, India

Ms. P.J. Pathak, Librarian, Institute for Plasma Research, India

Ms. Clelia De Palo, Associazione EURATOM-ENEA, Italy

Dr. G. Grosso, Instituto di Fisica del Plasma, Italy

Librarian, Naka Fusion Research Establishment, JAERI, Japan

Library, Laboratory for Complex Energy Processes, Institute for Advanced Study, Kyoto University, Japan

Research Information Center, National Institute for Fusion Science, Japan

Dr. O. Mitarai, Kyushu Tokai University, Japan

Dr. Jiangang Li, Institute of Plasma Physics, Chinese Academy of Sciences, People's Republic of China

Professor Yuping Huo, School of Physical Science and Technology, People's Republic of China

Library, Academia Sinica, Institute of Plasma Physics, People's Republic of China

Librarian, Institute of Physics, Chinese Academy of Sciences, People's Republic of China

Dr. S. Mirnov, TRINITI, Troitsk, Russian Federation, Russia

Dr. V.S. Strelkov, Kurchatov Institute, Russian Federation, Russia

Professor Peter Lukac, Katedra Fyziky Plazmy MFF UK, Mlynska dolina F-2, Komenskeho Univerzita, SK-842 15 Bratislava, Slovakia

Dr. G.S. Lee, Korea Basic Science Institute, South Korea

Institute for Plasma Research, University of Maryland, USA

Librarian, Fusion Energy Division, Oak Ridge National Laboratory, USA

Librarian, Institute of Fusion Studies, University of Texas, USA

Librarian, Magnetic Fusion Program, Lawrence Livermore National Laboratory, USA

Library, General Atomics, USA

Plasma Physics Group, Fusion Energy Research Program, University of California at San Diego, USA

Plasma Physics Library, Columbia University, USA

Alkesh Punjabi, Center for Fusion Research and Training, Hampton University, USA

Dr. W.M. Stacey, Fusion Research Center, Georgia Institute of Technology, USA

Dr. John Willis, U.S. Department of Energy, Office of Fusion Energy Sciences, USA

Mr. Paul H. Wright, Indianapolis, Indiana, USA 
The Princeton Plasma Physics Laboratory is operated by Princeton University under contract with the U.S. Department of Energy.

\author{
Information Services \\ Princeton Plasma Physics Laboratory \\ P.O. Box 451 \\ Princeton, NJ 08543
}

Phone: 609-243-2750

Fax: 609-243-2751

e-mail: pppl_info@pppl.gov

Internet Address: http://www.pppl.gov 